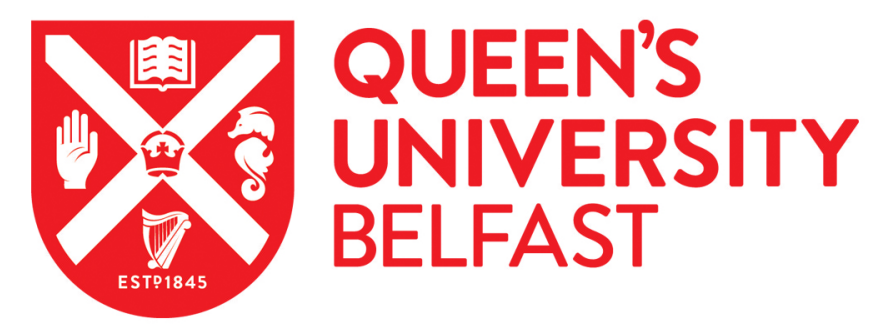

\title{
Prediction of Transonic LCOs using an Aeroelastic Harmonic Balance Method
}

Yao, W., \& Marques, S. (2014). Prediction of Transonic LCOs using an Aeroelastic Harmonic Balance Method. Paper presented at 44th AIAA Fluid Dynamics Conference, Atlanta, United States.

https://doi.org/10.2514/6.2014-2310

\section{Document Version:}

Peer reviewed version

\section{Queen's University Belfast - Research Portal:}

Link to publication record in Queen's University Belfast Research Portal

\author{
Publisher rights \\ Copyright @ 2014 by Weigang Yao and Simao Marques
}

\section{General rights}

Copyright for the publications made accessible via the Queen's University Belfast Research Portal is retained by the author(s) and / or other copyright owners and it is a condition of accessing these publications that users recognise and abide by the legal requirements associated with these rights.

\section{Take down policy}

The Research Portal is Queen's institutional repository that provides access to Queen's research output. Every effort has been made to ensure that content in the Research Portal does not infringe any person's rights, or applicable UK laws. If you discover content in the Research Portal that you believe breaches copyright or violates any law, please contact openaccess@qub.ac.uk. 


\title{
Prediction of Transonic Limit Cycle Oscillations using an Aeroelastic Harmonic Balance Method
}

\author{
W. Yao, S. Marques ${ }^{\dagger}$ \\ School of Mechanical and Aerospace Engineering \\ Queen's University Belfast, Belfast, UK, BT9 5AH
}

\begin{abstract}
This work proposes a novel approach to compute transonic Limit Cycle Oscillations using high fidelity analysis. CFD based Harmonic Balance methods have proven to be efficient tools to predict periodic phenomena. This paper's contribution is to present a new methodology to determine the unknown frequency of oscillations, enabling HB methods to accurately capture Limit Cycle Oscillations (LCOs); this is achieved by defining a frequency updating procedure based on a coupled CFD/CSD Harmonic Balance formulation to find the LCO condition. A pitch/plunge aerofoil and delta wing aerodynamic and respective linear structural models are used to validate the new method against conventional timedomain simulations. Results show consistent agreement between the proposed and timemarching methods for both LCO amplitude and frequency, while producing at least one order of magnitude reduction in computational time.
\end{abstract}

*Research Fellow, MAIAA

${ }^{\dagger}$ Lecturer, MAIAA 


\section{Nomenclature}

$\begin{array}{ll}\mathbf{A} & =\text { Harmonic Balance frequency domain matrix } \\ b, c & =\text { aerofoil semi-chord and chord, respectively } \\ \mathbf{D} & =\text { Harmonic Balance operator matrix } \\ E & =\text { energy } \\ \mathbf{E} & =\text { Tranformation matrix between frequency and time domains } \\ \mathbf{f} & =\text { fluid force acting on structure } \\ \mathbf{F}, \mathbf{G}, \mathbf{H} & =\text { convective fluxes for fluid equations } \\ h & =\text { plunge coordinate } \\ \mathbf{I} & =\text { HB residual } \\ \mathbf{K} & =\text { structure stiffness matrix } \\ \mathbf{L} & =\text { frequency updating figure of merit } \\ \mathbf{M} & =\text { structure mass matrix } \\ p & =\text { pressure } \\ \mathbf{R} & =\text { vector of fluid and/or structural equation residual } \\ t & =\text { time step } \\ U_{\infty} & =\text { free-stream velocity } \\ u, v, w & =\text { fluid cartesian velocity components } \\ V, V_{s} & =\text { reduced velocity and velocity index } \\ \mathbf{W} & =\text { vector of fluid unknowns } \\ \mathbf{x}, \mathbf{y} & =\text { vector of structural unknowns } \\ \alpha & =\end{array}$

\section{Introduction}

TNUSTRY standard practices to solve aeroelastic problems rely heavily upon linear aerodynamic theory. 1 This has well known limitations in the transonic regime and where other sources of aerodynamic nonlinearities are present (e.g., unsteady viscous flows), hence a clear need for physics based modelling tools has emerged as identified by Noll et al. ${ }^{1}$ When nonlinearities are present, aeroelastic instabilities can lead to oscillations that become limited and limit cycle oscillations are observed. This is a problem of considerable practical interest and is well documented for in-service aircraft. ${ }^{2,3}$ The presence of nonlinearities, either structural or aerodynamic, poses additional challenges both in terms of complexity and computational resources, by requiring higher-fidelity analysis. Such requirements can be exacerbated by the need to quantify the uncertainty due to unknown or variable parameters. Hence, several efforts have been made to address both issues of retaining the required level of fidelity to capture the relevant physics, while at the same time limiting the computational resources required for such analysis.

To overcome these restrictions, CFD (Computational Fluid Dynamics) methods can be coupled with CSD (Computational Structural Dynamics) in the time domain; however this type of analysis is used as a last resort tool due to the high computational cost. For several years, the research community has developed Reduced Order Models (ROM) to avoid the penalty of full order time domain analysis. Several methods have been proposed and used: Proper Orthogonal Decomposition (POD),${ }^{4,5}$ Volterra Series, ${ }^{6-8}$ Neural Networks, ${ }^{9}$ etc. Typically, ROM's lack generality and their application is restricted to a limited vicinity of the original parameters used in building the ROM.

To avoid such restrictions, model reduction based on centre manifold theory has shown the ability to predict LCOs without compromising the underlying physics of the problem. ${ }^{10,11}$ An alternative to ROM and full time domain analysis of aeroelastic oscillatory problems is to employ the non-linear Harmonic Bal- 
ance (HB) method. New Harmonic Balance methods have been developed for CFD time periodic flows; ${ }^{12,13}$ in such methods, the periodicity of the flow is exploited and represent time dependent flow variables as Fourier series and recast the problem in terms of Fourier coefficients. These methods have been successful in predicting unsteady flows efficiently in diverse applications: forced motions, ${ }^{14,15}$ helicopter rotors, ${ }^{16}$ turbomachinery. ${ }^{12,17,18}$ Thomas et al. extended the HB formulation to predict Limit Cycle Oscillations for fixed wing aircraft. ${ }^{3}$ Ekici and Hall further reduced the computational cost of predicting LCOs with HB methods, by proposing a one-shot method to analyze 1-DOF LCO in turbomachinery flows. ${ }^{17}$

As far as the authors are aware, only the method proposed by Thomas et al. ${ }^{3}$ has been able to predict LCOs for fixed wing aircraft using a CFD based HB formulation. This paper presents an alternative method to compute nonlinear aeroelastic instabilities (LCOs) using a coupled CFD-CSD Harmonic Balance formulation based on the Euler equations for fluid dynamics and linear structural models. The paper will first describe the details of the CFD and HB implementations, this will be followed by presenting a novel formulation for predicting LCOs amplitudes and frequencies of coupled aeroelastic systems. The method described will be first validated using experimental data from forced motion test cases, then the new Aeroelastic Harmonic Balance (A-HB) method will be used to predict LCOs in 2D and 3D test cases, results are assessed against time marching methods for accuracy and efficiency.

\section{Flow Solver}

The semi-discrete form of an arbitrary system for a system of conservation laws such as the the threedimensional Euler equations can be described as:

$$
\frac{\partial \mathbf{W}}{\partial t}=-R(\mathbf{W})
$$

where $R$ is the residual error of the steady-state solution:

$$
R=\frac{\partial \mathbf{F}}{\partial x}+\frac{\partial \mathbf{G}}{\partial y}+\frac{\partial \mathbf{H}}{\partial z}
$$

Here $\mathbf{W}$ is the vector containing the flow variables and $\mathbf{F}, \mathbf{G}, \mathbf{H}$ are the fluxes, which are given by:

$$
\mathbf{W}=\left[\begin{array}{c}
\rho \\
\rho u \\
\rho v \\
\rho w \\
\rho E
\end{array}\right], \mathbf{F}=\left[\begin{array}{c}
\rho u \\
\rho u u+p \\
\rho u v \\
\rho u w \\
u(\rho E+p)
\end{array}\right], \mathbf{G}\left[\begin{array}{c}
\rho v \\
\rho u v \\
\rho v v+p \\
\rho v w \\
v(\rho E+p)
\end{array}\right], \quad \mathbf{H}=\left[\begin{array}{c}
\rho w \\
\rho u w \\
\rho v w \\
\rho w w+p \\
w(\rho E+p)
\end{array}\right],
$$

The steady state solution of the Euler equation are obtained by marching the solution forward in time by solving the following discrete nonlinear system of equations:

$$
\frac{\mathbf{W}^{n+1}-\mathbf{W}^{n}}{\Delta t}=-\mathbf{R}^{n}
$$

To discretize the residual convective terms a Roe flux function ${ }^{19}$ together with MUSCL interpolation is used, ${ }^{20}$ the Van Albada limiter is used to obtaion $2^{\text {nd }}$ order accuracy. The nonlinear system of algebraic equations represented by eq. (4) is solved by an explicit, 4-stage, Runge-Kutta method.

\section{Harmonic Balance Formulation}

As discussed in the introduction, several authors have demonstrated the suitability of HB methods as an alternative to time marching CFD formulations for periodic flow problems. To obtain the HB version of the flow solver, we follow the methodology presented by Badcock and Woodgate, ${ }^{14}$ which is summarised next. Consider the semidiscrete form as a system of ordinary differential equations

$$
\mathbf{I}(t)=\frac{d \mathbf{W}(t)}{d t}+\mathbf{R}(t)=0
$$


The solution of $\mathbf{W}$ and $\mathbf{R}$ in eq.(5) can be approximated to be a truncated Fourier series of $N_{H}$ harmonics with a fundamental frequency $\omega$ :

$$
\begin{aligned}
\mathbf{W}(t) & \approx \hat{\mathbf{W}}_{0}+\sum_{n=1}^{N_{H}}\left(\hat{\mathbf{W}}_{2 n-1} \cos (n \omega t)+\hat{\mathbf{W}}_{2 n} \sin (n \omega t)\right) \\
\mathbf{R}(t) & \approx \hat{\mathbf{R}}_{0}+\sum_{n=1}^{N_{H}}\left(\hat{\mathbf{R}}_{2 n-1} \cos (n \omega t)+\hat{\mathbf{R}}_{2 n} \sin (n \omega t)\right)
\end{aligned}
$$

Hence, eq.(5) can also be approximated by a truncated Fourier series,

$$
\mathbf{I}(t) \approx \hat{\mathbf{I}}_{0}+\sum_{n=1}^{N_{H}}\left(\hat{\mathbf{I}}_{2 n-1} \cos (n \omega t)+\hat{\mathbf{I}}_{2 n} \sin (n \omega t)\right)
$$

which results in the following system of equations

$$
\begin{aligned}
\hat{\mathbf{I}}_{0} & =\hat{\mathbf{R}}_{0} \\
\hat{\mathbf{I}}_{2 n-1} & =\omega n \hat{\mathbf{W}}_{2 n}+\hat{\mathbf{R}}_{2 n-1} \\
\hat{\mathbf{I}}_{2 n} & =-\omega n \hat{\mathbf{W}}_{2 n-1}+\hat{\mathbf{R}}_{2 n}
\end{aligned}
$$

which results in a system of $\left(2 N_{H}+1\right)$ equations for the Fourier coefficients that can be expressed in matrix form as

$$
\omega \mathbf{A} \hat{\mathbf{W}}+\hat{\mathbf{R}}=0
$$

where $\mathbf{A}$ is given by:

$$
\mathbf{A}=\left[\begin{array}{llll}
0 & & & \\
& \mathbf{J}_{1} & & \\
& & \ddots & \\
& & & \mathbf{J}_{N_{H}}
\end{array}\right]_{\left(2 N_{H}+1\right) \times\left(2 N_{H}+1\right)}, \mathbf{J}=n\left[\begin{array}{cc}
0 & 1 \\
-1 & 0
\end{array}\right], n=1,2, \ldots, N_{H}
$$

To overcome the difficulties in expressing the Fourier coefficient in $\hat{\mathbf{R}}$ as functions of $\hat{\mathbf{W}}$, Hall et al. ${ }^{12}$ proposed to cast the system of equations back in the time domain, where the flow variables and residual solutions are split into $\left(2 N_{H}+1\right)$, discrete, equally spaced intervals over the period $T=\frac{2 \pi}{\omega}$.

$$
\mathbf{W}_{h b}=\left[\begin{array}{c}
\mathbf{W}\left(t_{0}+\Delta t\right) \\
\mathbf{W}\left(t_{0}+2 \Delta t\right) \\
\vdots \\
\mathbf{W}\left(t_{0}+T\right)
\end{array}\right], \quad \mathbf{R}_{h b}=\left[\begin{array}{c}
\mathbf{R}\left(t_{0}+\Delta t\right) \\
\mathbf{R}\left(t_{0}+2 \Delta t\right) \\
\vdots \\
\mathbf{R}\left(t_{0}+T\right)
\end{array}\right]
$$

It is possible to define a transformation matrix, $\mathbf{E}$ that relates the frequency domain variables to their HB time domain counterpart ${ }^{12}$

$$
\hat{\mathbf{W}}=\mathbf{E W}_{h b} \quad \hat{\mathbf{R}}=\mathbf{E R}_{h b}
$$

Substituting the terms in eq.(15) in eq.(12), it becomes:

$$
\begin{gathered}
\omega \mathbf{A} \hat{\mathbf{W}}+\hat{\mathbf{R}}=0=\omega \mathbf{A} \mathbf{E} \mathbf{W}_{h b}+\mathbf{E R}_{h b}=\omega \mathbf{E}^{-1} \mathbf{A} \mathbf{E} \mathbf{W}_{h b}+\mathbf{R}_{h b}= \\
=\omega \mathbf{D W} \mathbf{W}_{h b}+\mathbf{R}_{h b}=0
\end{gathered}
$$

where $\mathbf{D}=\mathbf{E}^{-1} \mathbf{A E}$, the elements in matrix $\mathbf{D}$ are given by:

$$
\mathbf{D}_{i, j}=\frac{2}{2 N_{H}+1} \sum_{k=1}^{N_{H}} k \sin \left(\frac{2 \pi k(j-i)}{2 N_{H}+1}\right)
$$


To solve eq.(16) a pseudo time step of the form is introduced:

$$
\frac{d \mathbf{W}_{h b}}{d \tau}+\omega \mathbf{D W}_{h b}+\mathbf{R}_{h b}=0
$$

To solve eq.(18), any steady-state CFD time marching method can be used. In this work an explicit 4-stage Runge-Kutta scheme is employed. The solution to eq.(18) corresponds to the flow solution at $2 N_{H}+1$ equally spaced time sub levels. The Fourier coefficients can be obtained by applying transformation matrix $\mathbf{E}$, and the flow field at any time level can be recovered by using Fourier expansions on the flow variables.

\section{Aeroelastic Formulation}

Consider a generic dynamic system without damping, whose behaviour can be described using the equation of motion given by:

$$
\mathbf{M} \ddot{\mathbf{x}}+\mathbf{K x}=\mathbf{f}
$$

where $\mathbf{M}, \mathbf{K}$, respectively, represent the mass and stiffness of the system and $\mathbf{f}$ is an external force (in this work, this will be the aerodynamic force, $\mathbf{f}=\mathbf{f}(\mathbf{W}, \omega, \mathbf{x})$. This equation can be transformed into a state-space form, giving:

$$
\dot{\mathbf{y}}=\mathbf{A}_{\mathbf{s}} \mathbf{y}+\mathbf{B}_{\mathbf{s}} \mathbf{f}
$$

where:

$$
\mathbf{A}_{\mathbf{s}}=\left[\begin{array}{cc}
0 & \mathbf{I} \\
\mathbf{M}^{-1} \mathbf{K} & 0
\end{array}\right], \quad \mathbf{B}_{\mathbf{s}}=\left[\begin{array}{c}
0 \\
\mathbf{M}^{-1}
\end{array}\right], \quad \mathbf{y}=\left[\begin{array}{c}
\mathbf{x} \\
\dot{\mathbf{x}}
\end{array}\right]
$$

Equation (20) has a similar form to the flow equations, hence it can be solved using the Harmonic Balance method describe in the previous section, resulting in the following HB format of eq.(20):

$$
\omega \mathbf{D} \mathbf{y}_{h b}=\mathbf{A}_{s} \mathbf{y}_{h b}+\mathbf{B}_{s} \mathbf{f}_{h b}
$$

where $\mathbf{D}$ is the same HB operator described in eq.(17). Equation (22) can be solved using the same pseudo time technique showed before, leading to the following system of equations: ${ }^{17}$

$$
\frac{d \mathbf{y}_{h b}}{d \tau}+\omega \mathbf{D y}_{h b}+\left(\mathbf{A}_{s} \mathbf{y}_{h b}+\mathbf{B}_{s} \mathbf{f}_{h b}\right)=0
$$

Equation (18) together with eq.(23) represent the nonlinear coupled aeroelastic system; when solving the aeroelastic system of equations, at each iteration, the generalized aerodynamic forces are computed using eq.(18), which will feed into eq.(23). The solution from eq.(23) will provide new generalized displacement and velocities to eq.(18).

\section{A. Prediction of Limit-Cycle Oscillations}

The prediction of LCO depends on determining a solution vector for $[\omega, \mathbf{y}]$ (the subscript $h b$ is dropped for simplicity), that satisfies both the structural governing equation eq.(23) and eq.(18). If the LCO frequency, $\omega$, is given beforehand, then the coupling itself becomes a fixed point iteration process which is extensively used for static aero-elastic problems in its time domain counterpart. ${ }^{15}$ A straightforward method to search for solutions for LCO is to employ a Newton-Raphson method. ${ }^{21}$ From eq.(22) define the following residual:

$$
\mathbf{R}(\omega, \mathbf{y})=\omega \mathbf{D} \mathbf{y}-\left(\mathbf{A}_{s} \mathbf{y}+\mathbf{B}_{s} \mathbf{f}\right)
$$

Applying the Newton-Raphson method to eq.(24), we get:

$$
\left[\begin{array}{l}
\omega \\
\mathbf{y}
\end{array}\right]_{n+1}=\left[\begin{array}{c}
\omega \\
\mathbf{y}
\end{array}\right]_{n}-\lambda \mathbf{J}^{-1} \mathbf{R}\left(\omega_{n}, \mathbf{y}_{n}\right)
$$

Where $\mathbf{J}$ is the Jacobian of $\mathbf{R}$ with respect to the solution vector $[\omega, \mathbf{y}]$; the expensive part is to approximate the derivatives of $\mathbf{f}$ with respect to $[\omega, \mathbf{y}]$ by finite differencing. If the number of harmonics used in eq.(18) is 
$N_{H}$, the structural degree-of-freedom (DOF) is $N_{s}$, then the fluid system needs to be evaluated $\left(N_{s}\left[\left(2 N_{H}+\right.\right.\right.$ $1) \times 2]+1$ ) times to form $\mathbf{J}$. As long as the initial guess is good enough, the Newton-Raphson method usually achieves converged solutions rapidly and efficiently. Thomas et al. ${ }^{21}$ demonstrated the effectiveness of this method for LCO prediction. For higher number of harmonics and structural DOF, the computational cost of building the Jacobian $\mathbf{J}$ itself is significant, ${ }^{14}$ and thus makes the Newton-Raphson method less attractive. Ekici and Hall ${ }^{17}$ developed a one-shot determination approach by advancing the structural and fluid system to convergence at the same time, with frequency updating. The authors demonstrate the overall computational cost is lower than the Newton-Raphson method for a 2D turbomachinery problem, with a single DOF structural governing equation. As shown before, a pseudo time marching strategy can be adopted to solve eq.(23): ${ }^{17}$

$$
\frac{\partial \mathbf{y}}{\partial \tau}+\omega \mathbf{D} \mathbf{y}-\left(\mathbf{A}_{s} \mathbf{y}+\mathbf{B}_{s} \mathbf{f}\right)=0
$$

Where $\tau$ is the pseudo-time step size, hence the convergence of eq.(23) highly depends on this time step size; in other words, $\tau$ should be large enough to march eq.(23) rapidly to convergence and at the same time ensure numerical stability. However, unlike the fluid system where local time step size can be used, for the HB system in eq.(23), a constant value is required. However, as the structural DOF increases, a significant deterioration of the convergence rate for eq.(23) was observed, even when using an implicit algorithm formulation.

To determine the LCO condition using eq.23, the frequency updating can be achieved by minimizing the $L_{2}$ norm of the residual $\mathbf{R}$ of eq.(24). ${ }^{17}$ First, define a figure of merit, in this case:

$$
\mathbf{L}_{n}=\frac{1}{2} \mathbf{R}^{T} \mathbf{R}=\frac{1}{2}\left[\omega \mathbf{D y}-\left(\mathbf{A}_{s} \mathbf{y}+\mathbf{B}_{s} \mathbf{f}\right)\right]^{T}\left[\omega \mathbf{D y}-\left(\mathbf{A}_{s} \mathbf{y}+\mathbf{B}_{s} \mathbf{f}\right)\right]
$$

Then derive the first order derivative with respect to $\omega$ :

$$
\frac{\partial \mathbf{L}_{n}}{\partial \omega}=(\mathbf{D y})^{T}\left[\omega \mathbf{D} \mathbf{y}-\left(\mathbf{A}_{s} \mathbf{y}+\mathbf{B}_{s} \mathbf{f}\right)\right]
$$

For a given vector $[\mathbf{y}, \mathbf{f}]$, the frequency can be solved directly by manipulating small matrices. It is worth noting that eq.(27) is derived with the force vector $\mathbf{f}$ frozen. The results presented below demonstrate that this assumption makes updating the frequency difficult and slow for multiple DOF systems.

\section{B. Proposed Approach for LCO Predictions}

Inspired by the results of Blanc et al. ${ }^{15}$ the present work aims to transform this LCO prediction problem into a fixed point algorithm with frequency updating. As before, the basic idea is to solve the linear eq.(22) for a given combination of $[\omega, \mathbf{f}]$, then transfer the displacement back to the fluid system. As in eq.(26), the frequency is updated by minimizing the residual $\mathbf{R}$ but, critically, without freezing the aerodynamic forces f, leading to:

$$
\frac{\partial \mathbf{L}_{n}}{\partial \omega}=\left(\mathbf{D} \mathbf{y}-\mathbf{B}_{s} \frac{\partial \mathbf{f}}{\partial \omega}\right)^{T}\left[\omega \mathbf{D y}-\left(\mathbf{A}_{s} \mathbf{y}+\mathbf{B}_{s} \mathbf{f}\right)\right]
$$

If the frequency $\omega$ is not at the LCO condition, the residual $\mathbf{R}$ for the displacement is not able to converge. Therefore, the idea is to update frequency every $n_{i}$ iterations.

The new algorithm is represented in Algorithm 1. When compared to the standard fixed point algorithm described by Blanc et al., ${ }^{15}$ the new algorithm introduces some extra computational effort to compute the gradient of the aerodynamic force with respect to the frequency. However, the frequency is only updated every $n_{i}$ iterations (typically every 10-15 iterations) and the perturbation is sufficiently small, minimizing the computational cost.

\section{Results}

\section{A. Code Validation}

The AGARD CT $6^{22}$ case is chosen to validate the HB-CFD code, this case is a prescribed pitching NACA $64 A 010$ aerofoil, the flow condition and motion parameters are listed in Table 1. 


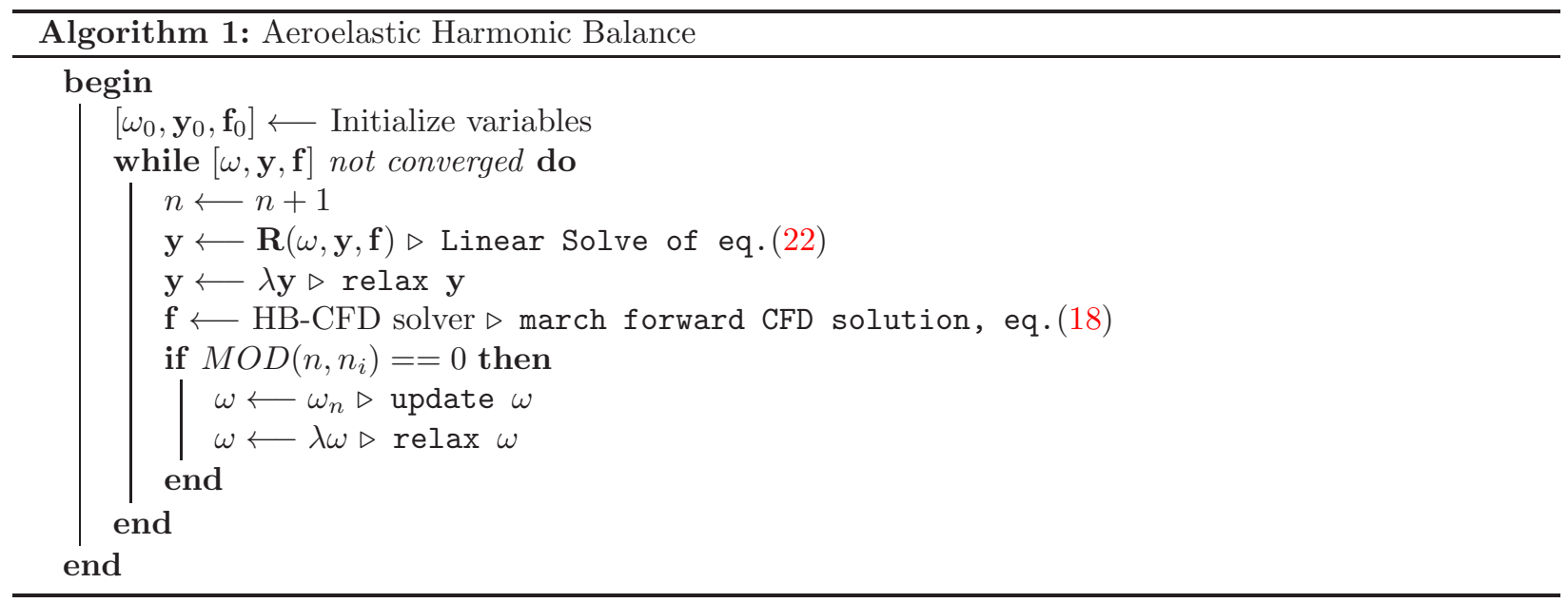

\begin{tabular}{cccccc}
\hline \hline Case & $M_{\infty}$ & $\alpha_{m}$ & $\alpha_{0}$ & $\mathrm{k}$ & $x_{m}$ \\
\hline CT6 & 0.796 & 0 & 1.01 & 0.202 & 0.25 \\
\hline \hline
\end{tabular}

Table 1. CT6 Case Parameters

An O-type grid as the one shown in fig.1 was used. A grid convergence study is carried out in terms of lift and moment coefficients. Three harmonics are used for all the results shown in fig.2. It is clearly shown in fig. 2 that a $61 \times 21$ grid is sufficient to capture the details of the unsteady lift and moment coefficients. The experimental data is also included for comparison. The lift coefficient agrees well with the data generally, however, large discrepancies are observed for the moment coefficient. This disagreement is consistent with results reported in the literature $^{23,24}$ and remains an enigma. Additionally, a convergence study concerning the number of harmonics required to accurately recreate the periodic forces is also carried out and results are shown in fig.3, which demonstrate that three harmonics are sufficient to obtain converged lift and pitching moment coefficients predictions. Therefore, the $61 \times 21$ grid and three harmonics are adopted for the following LCO study.

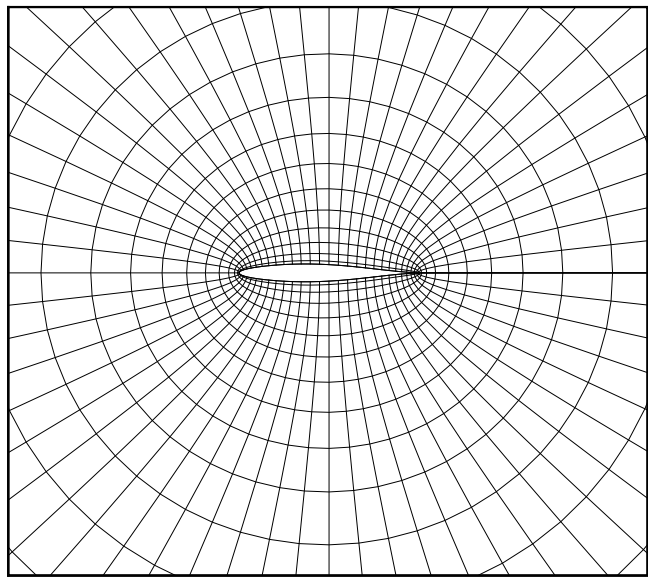

Figure 1. O-grid over NACA $64 A 010$ aerofoil However, it is worth noting that this systematic convergence study is carried out only based on AGARD CT6 case, a subsequent convergence study with respect to LCO amplitude and frequency is also necessary. 


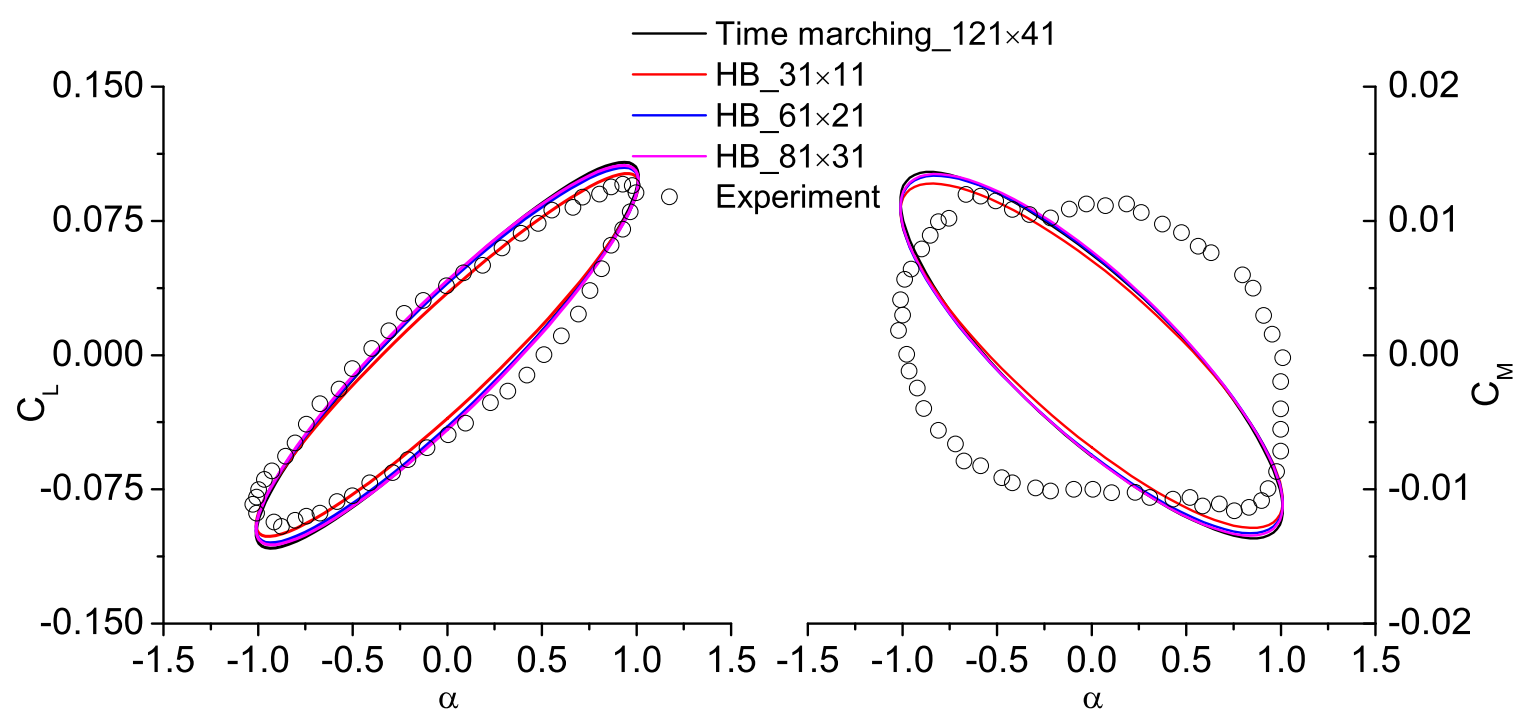

Figure 2. Grid Convergence Study for CT6 Case

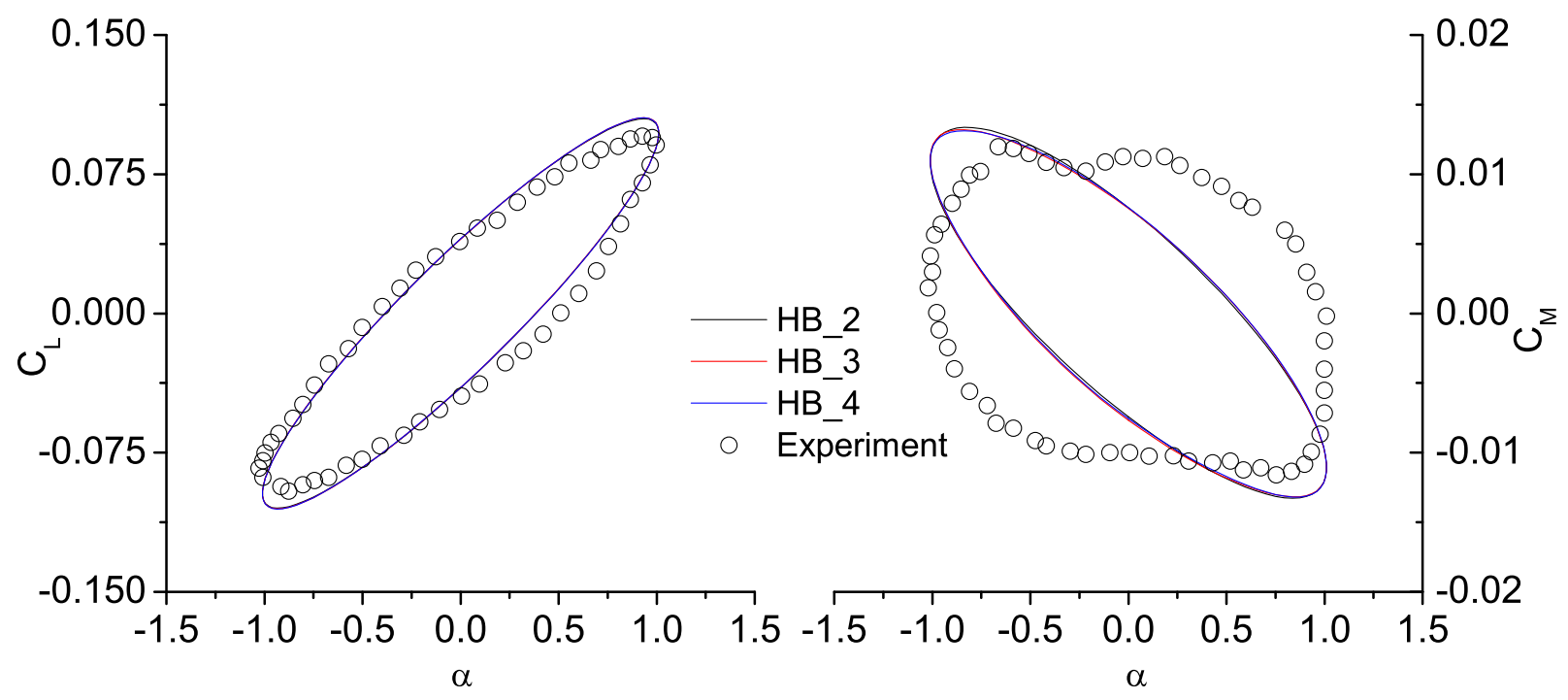

Figure 3. Number of Harmonics Convergence Study for CT6 Case 


\section{B. LCO Predictions}

In order to validate the coupling approach proposed in this paper, a pitch/plunge symmetric NACA $64 A 010$ aerofoil, shown in fig.4, is used to assess its efficiency and effectiveness. The equations of motion for this pitch-plunge aerofoil problem can be found in ref.: ${ }^{21}$

$$
\begin{aligned}
m \ddot{h}+S_{\alpha} \ddot{\alpha}+K_{h} & =-q_{\infty} c C_{l} \\
S_{\alpha} \ddot{h}+I_{\alpha} \ddot{\alpha}+K_{\alpha} & =q_{\infty} c^{2} C_{m}
\end{aligned}
$$

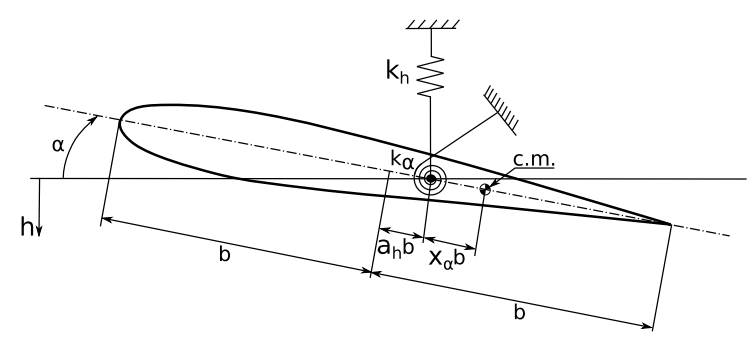

where $m$ is the mass of the aerofoil, $S_{\alpha}, I_{\alpha}$ are the first and second moment of inertia about the elastic axis, respectively; $q_{\infty}$ represents the dynamic pressure and

Figure 4. Diagramatic representation of pitch/plunge, two-degree-of-freedom aerofoil

$C_{l}, C_{m}$ are the lift and pitching moment coefficient.

Following Thomas et al.,${ }^{21}$ the non-dimensional form of eq.(29) and (30) is given by

$$
\mathbf{M} \ddot{\mathbf{y}}+\frac{1}{V^{2}} \mathbf{K y}=\frac{4}{\pi \mu} \mathbf{f}
$$

where

$$
\mathbf{M}=\left[\begin{array}{cc}
1 & x_{\alpha} \\
x_{\alpha} & r_{\alpha}^{2}
\end{array}\right], \mathbf{K}=\left[\begin{array}{cc}
\left(\frac{\omega_{h}}{\omega_{\alpha}}\right)^{2} & 0 \\
0 & r_{\alpha}^{2}
\end{array}\right], \mathbf{f}=\left[\begin{array}{c}
-C_{l} \\
2 C_{m}
\end{array}\right], \quad \mathbf{y}=\left[\begin{array}{c}
\frac{h}{b} \\
\alpha
\end{array}\right], \quad V=\frac{U_{\infty}}{\sqrt{\mu \omega_{\alpha} b}}
$$

and the structural parameters chosen are:

\begin{tabular}{lc}
\hline \hline Static unbalance, $x_{\alpha}=S_{\alpha} / m b$ & 0.25 \\
Radius of gyration about elastic axis, $r_{\alpha}^{2}=I_{\alpha} / m b^{2}$ & 0.75 \\
Frequency ratio, $\omega_{h} / \omega_{\alpha}$ & 0.5 \\
Mass ratio, $\mu=m / \pi \rho_{\infty} b^{2}$ & 75 \\
\hline \hline
\end{tabular}

Table 2. Pitch/Plunge Aerofoil Parameters

Besides the above parameters, the Mach number and the initial angle of attack are set to 0.8 and $0^{\circ}$, respectively, the aeroelastic axis distance from the centre chord is: $a_{h} / b=-0.6$. The velocity index is used to set different conditions (dynamic pressure, altitude, etc) for the analysis. For a given value of the velocity index, eq.(31) is solved using the procedure described in the section B, determining the final amplitude and frequency of the periodic motion. The velocity index is defined as:

$$
V_{s}=\frac{U_{\infty}}{b \omega_{\alpha} \sqrt{\mu}}
$$

Following the proposed algorithm 1, the Aeroelastic-HB solver needs to initialize the structural and fluid system's $[\omega, \mathbf{y}, \mathbf{f}]$. The critical part is how to determine the initial frequency. Once the frequency is determined the coupling algorithm becomes a fixed point problem, leading to a very fast convergence of the coupled problem, as illustrated by Blanc et al., ${ }^{15}$ even over relative large margins of the initial values of the displacement $\mathbf{y}$. A fairly good initial $\omega$ ought to be a frequency near the flutter condition. Volterra series along with the ERA (Eigensystem Realization Algorithm) method, as described by Silva ${ }^{6}$ is employed to construct a linear CFD

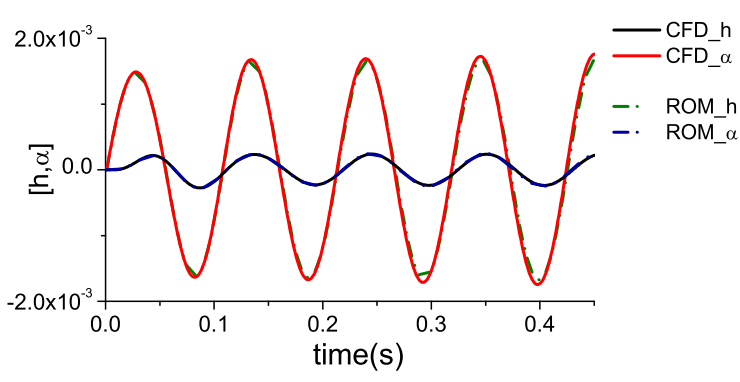

Figure 5. Pitch/plunge aerofoil flutter response 
model coupled with the structural model to form a $24^{\text {th }}$

ROM. The resultant CFD and ROM prediction for this

aerofoil case is shown in fig.5. The flutter condition, using the reduced frequency is $\left[\kappa, V_{s}\right]=[0.1089,0.693]$.

Because of the nonlinear aerodynamic force, it is expected that at conditions above the corresponding flutter point $V_{s}$, the oscillation to become bounded and form a finite amplitude oscillation or LCO. Without loss of generality, the starting point is chosen to be $\left[\kappa, V_{s}\right]=[0.1,0.725]$, meaning an LCO at $V_{s}=0.725$ needs to be computed with an initial frequency guess $\kappa=0.1$ which is very close to the flutter condition. As described in the coupling algorithm, the fluid system is initialized with $\left[\kappa, V_{s}\right]=[0.1,0.725]$ and an initial displacement given by: $\mathbf{y}=[0.2,0.02 \mathrm{rad}]^{T}$. Figure 6 shows the fluid convergence history for the first three sub-levels of the A-HB formulation. The residual drops monotonically, note that once the residual is below the level of $10^{-5}$, the fluid force is no longer varying significantly.

Snapshots of the transonic pressure field, corresponding to the seven intervals used when solving for 3 harmonics, are shown in fig.7. Here the shock-wave motion captured by the A-HB method is clearly visible, as well as its motion throughout the plunging and pitching motions. The main purpose of this test case is to evaluate the solver, particularly the frequency updating procedure. Figure 8-a) shows the frequency converging rapidly in fewer than 500 iterations for $V_{s}=0.725$ to a value of $\kappa=0.1055$, approximately $5 \%$ above the initial value. Once the LCO is captured, then the intuitive way to compute LCO at different conditions is to use $[\omega, \mathbf{y}, \mathbf{f}]_{V_{s}=0.725}$ as the initial guess. Therefore the frequency computed at $V_{s}=0.725$ is used for LCOs predictions at $V_{s}=0.8$; the initial perturbation remains the same, $\mathbf{y}=[0.2,0.02 \mathrm{rad}]^{T}$. Results are illustrated in fig.8-b), it takes fewer than 400 iterations for the frequency to converge at the new velocity index. Both these results converge to LCO

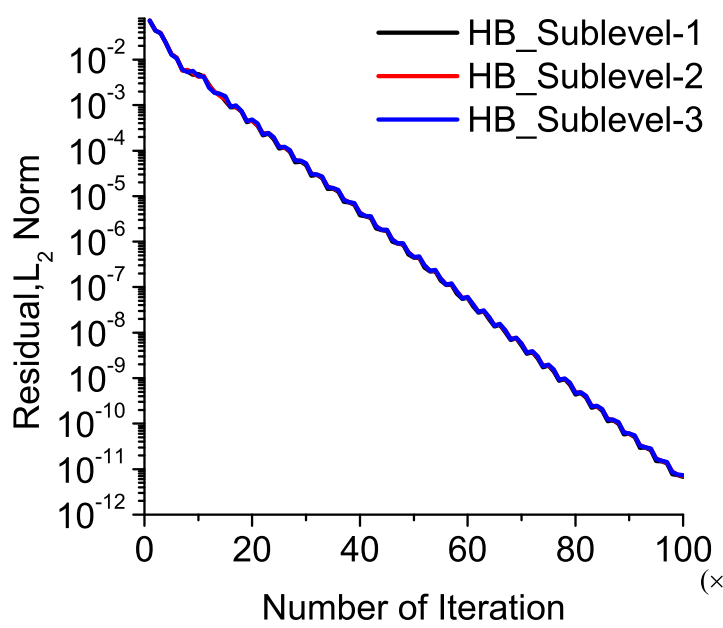

Figure 6. Convergence history of first three sublevels frequency values computed using conventional time marching methods, noted in the graphs as the fixed $\omega_{-} L C O$.

The performance of the updating procedures using either eq.(27) or eq.(28) are assessed in fig.8-c) and 8-d). The results suggest that by including the aerodynamic force gradient with respect to $\omega$, the overall computational cost is decreased; both LCO frequency and amplitude converge significantly faster using the new procedure proposed in this paper. Figure 9 shows the displacement convergence when using a fixed value for $\omega$ and when solving for $\omega$ using eq.(28). The displacement converges faster when $\omega$ is fixed, as expected. It also indicates once the frequency converges, the displacement should converge very rapidly.

This test case is also used to assess the impact of retaining a different number of harmonics on the LCO results. Figure 10 shows the position-velocity diagram for both plunge and pitch variables, using $1-4$ harmonics. The LCO cycle shown by the continuous line, is reconstructed by the A-HB solution using 90 points. The difference between retaining 2, 3 or 4 harmonics is minimal. A detailed example of the reconstruction cycle by the A-HB for $V_{s}=0.8$ is given in fig.11, in this case retaining two harmonics slightly under-predicts the motion's amplitudes. A comparison between the LCO solutions, using position-velocity diagrams, obtained by the A-HB method (using 3 harmonics) and time marching results is given in fig.12 and 13, showing good level of agreement.

The LCO amplitude growth predicted by the A-HB method, retaining 3 harmonics, is compared against a time marching method in fig.14, showing consistent results for all conditions analysed. The effects of increasing the velocity index is shown in detail in fig.14-a)-b); increasing the velocity index produces a supercritical LCO. The impact of increasing the frequency ratio of the normal modes, shown in fig.14-c)d), also produces a supercritical LCO, however the initial sensitivity of the amplitude to this parameter is higher. In both parametric changes and for higher amplitudes, the A-HB shows excellent agreement with time-marching results. Furthermore, the results for the conditions tested here are consistent with the equivalent case reported in Thomas et al. ${ }^{21}$ 


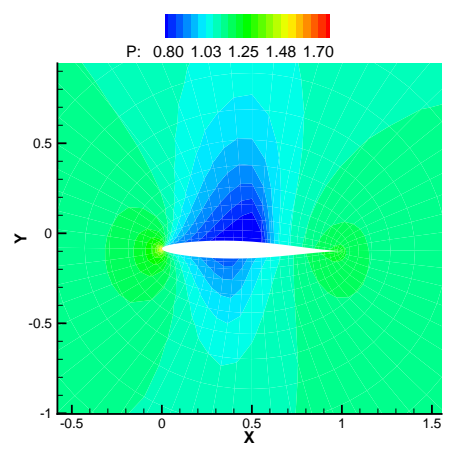

(a) Sub-level 1

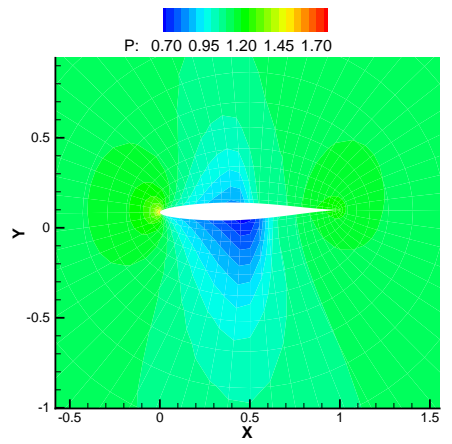

(d) Sub-level 4

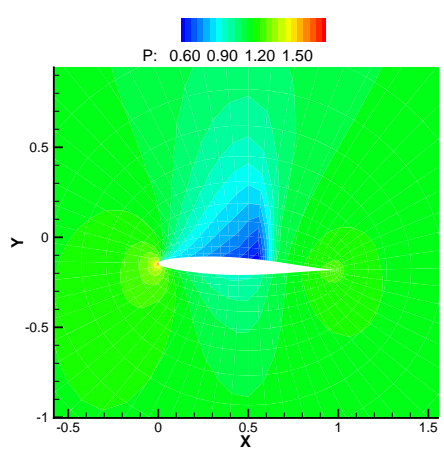

(b) Sub-level 2

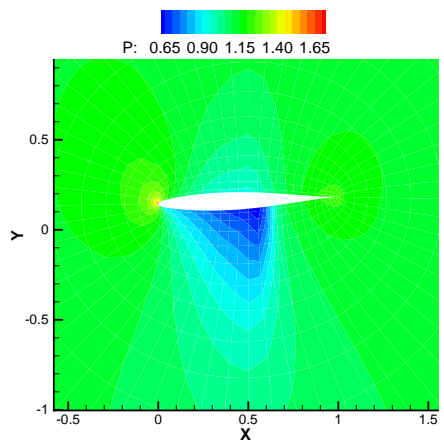

(e) Sub-level 5

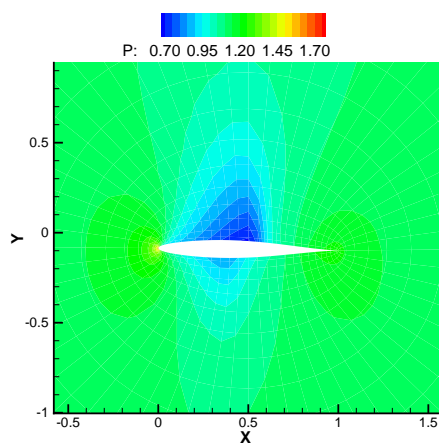

(g) Sub-level 7

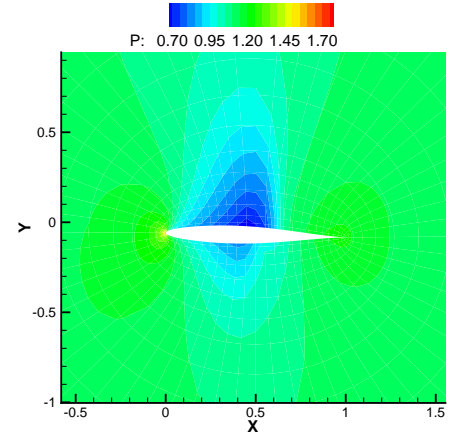

(c) Sub-level 3

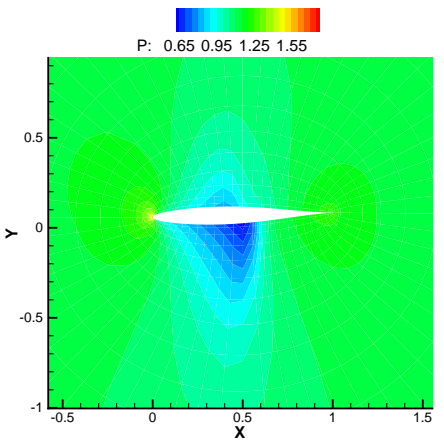

(f) Sub-level 6

Figure 7. Pressure field snapshots during LCO cycle, $V_{s}=0.725$ 

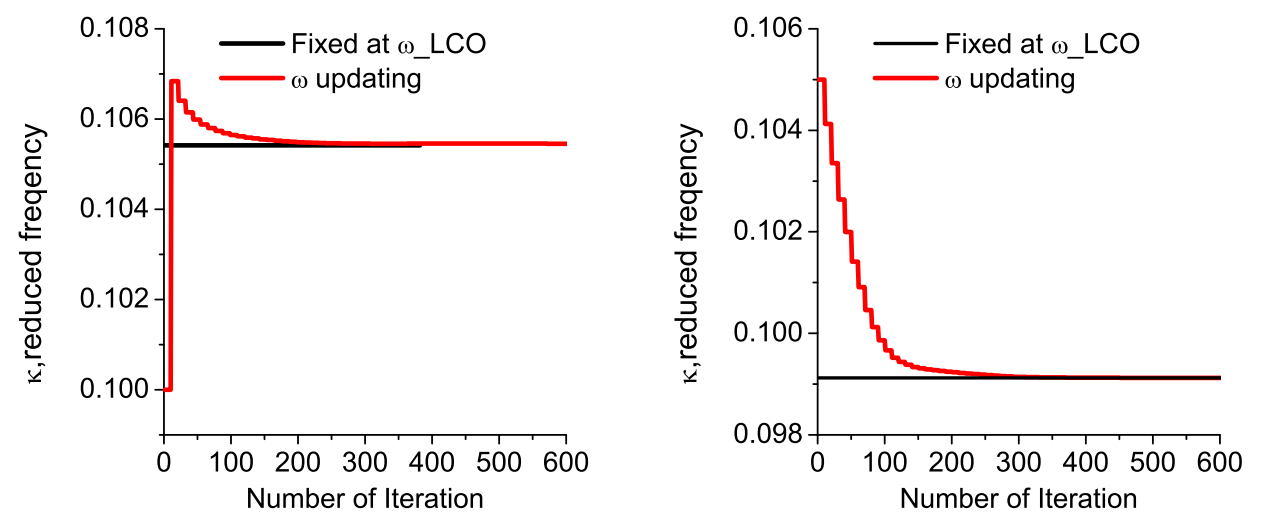

(a) Frequency Convergence $-V_{s}=0.725$

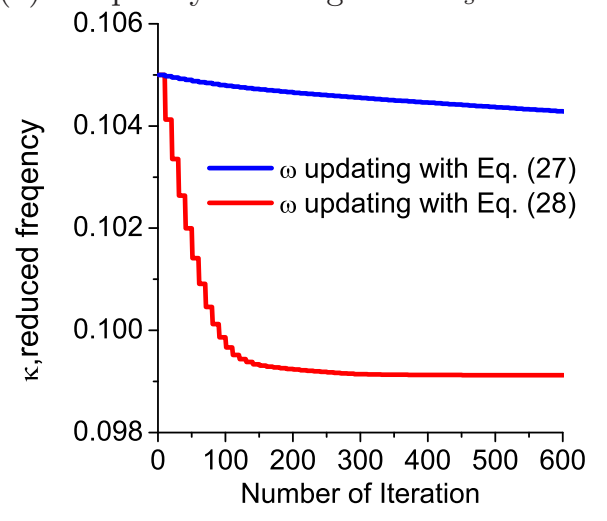

(b) Frequency Convergence - $V_{s}=0.80$

(c) Frequency Updating - $V_{s}=0.8$

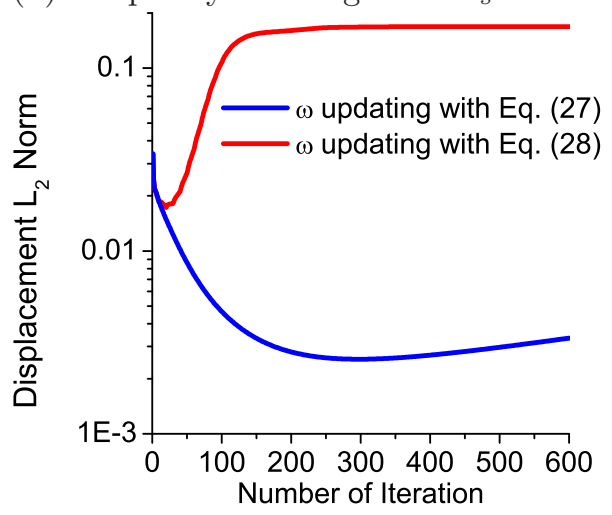

(d) Displacement Convergence - $V_{s}=0.8$

Figure 8. A-HB Frequency and Amplitude LCO convergence

The increase in the number of harmonics retained to solve the unsteady problem can have a significant impact on the computational effort required. Table 3 includes the time necessary to reach convergence for time marching and the A-HB solutions. To ensure the cycle was well resolved, a time step convergence study was performed, it was found that the amplitude reached convergence for time steps smaller than $4 \times 10^{-5}$. When using 1 harmonic the A-HB is over one order of magnitude faster than the time-marching approach, whereas when retaining 4 harmonics, the solution requires $33 \%$ of the effort required by the time-marching solver.

\begin{tabular}{ccc}
\hline \hline & Wall Clock $[s]$ & Speed-Up \\
\hline Time Marching & 3990 & 1.0 \\
1 Harmonic & 282 & 14.1 \\
2 Harmonics & 480 & 8.3 \\
3 Harmonics & 1050 & 3.8 \\
4 Harmonics & 1224 & 3.3 \\
\hline \hline
\end{tabular}

Table 3. Run times for aerofoil test case, $V_{s}=0.80$, with different number of harmonics per cycle 


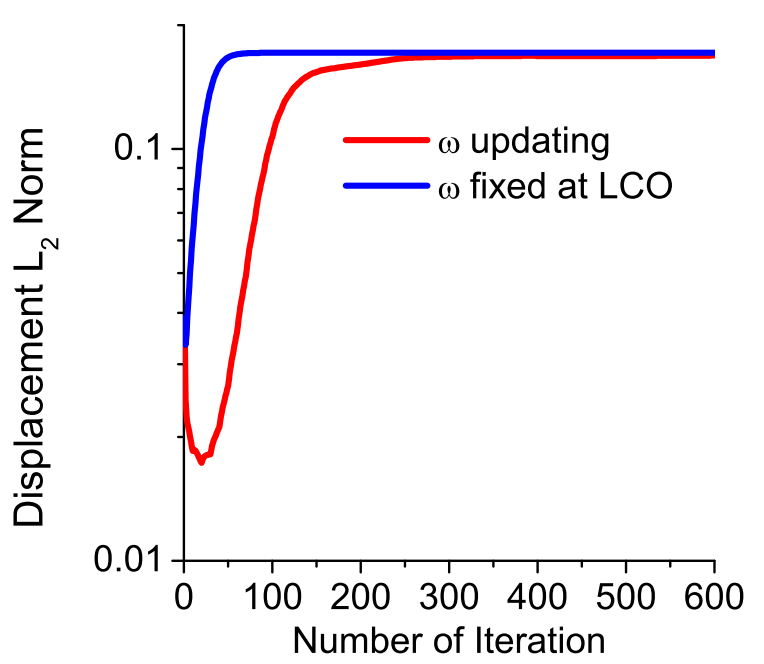

(a) - Displacement

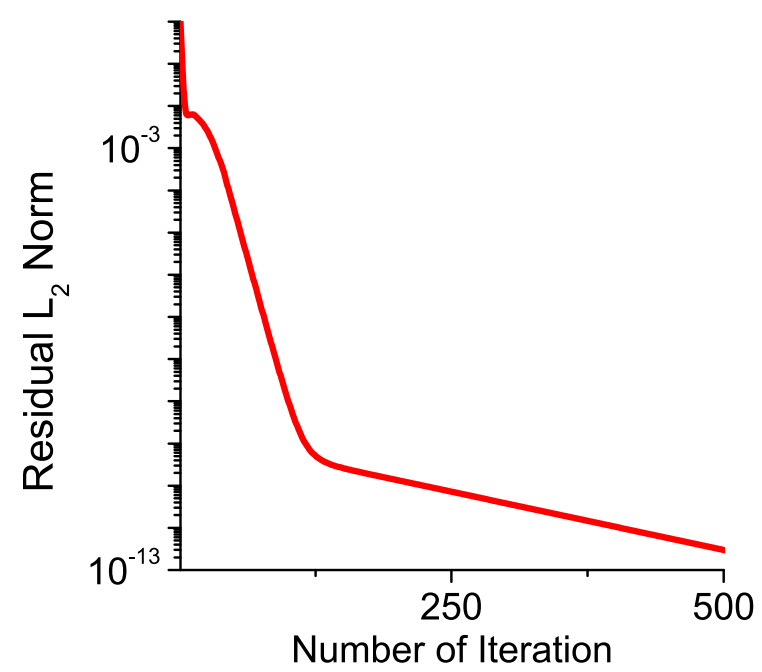

(b) Structural Residual

Figure 9. Convergence of Aeroelastic system, fixed $\omega-V_{s}=0.80$

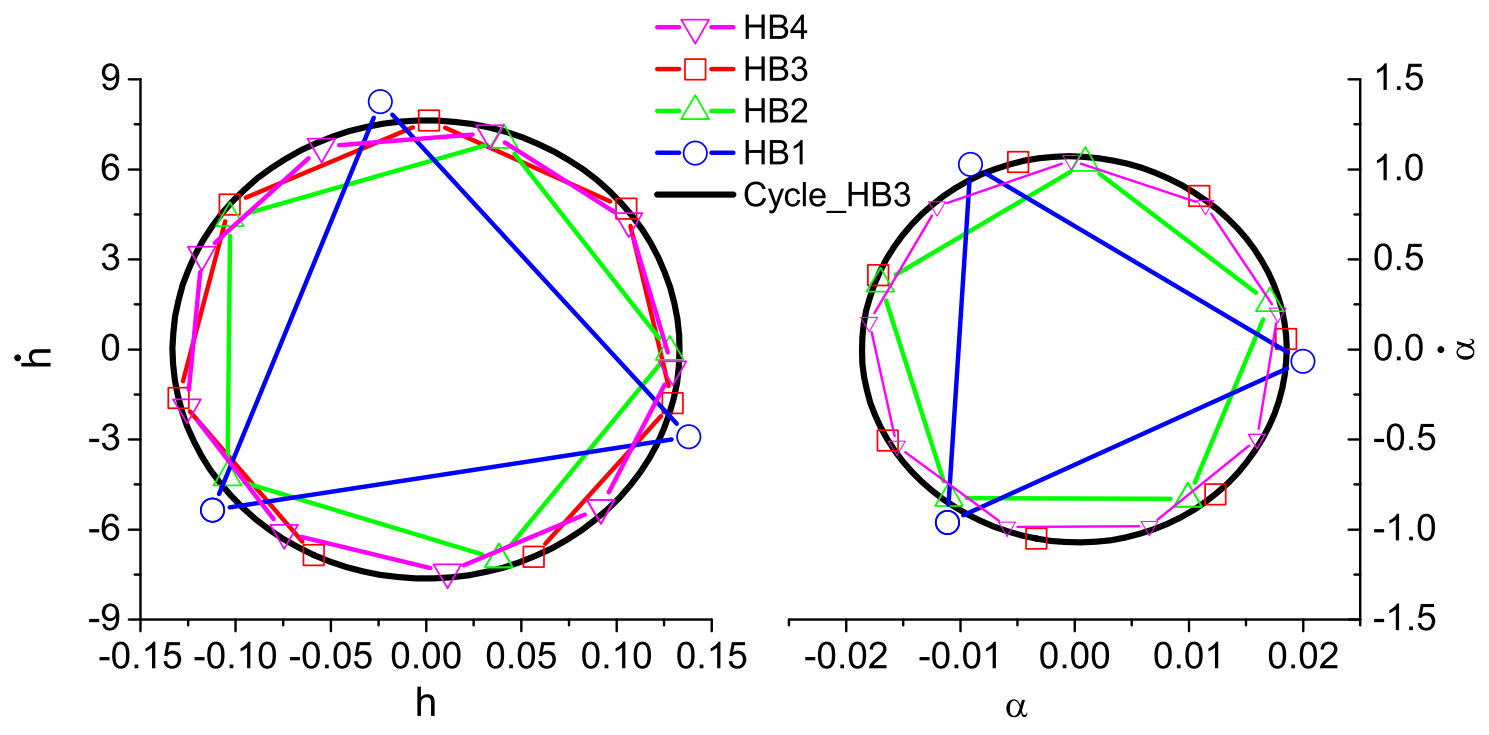

Figure 10. Position - Velocity diagram at LCO condition - $V_{s}=0.725$ - Harmonic Convergence 


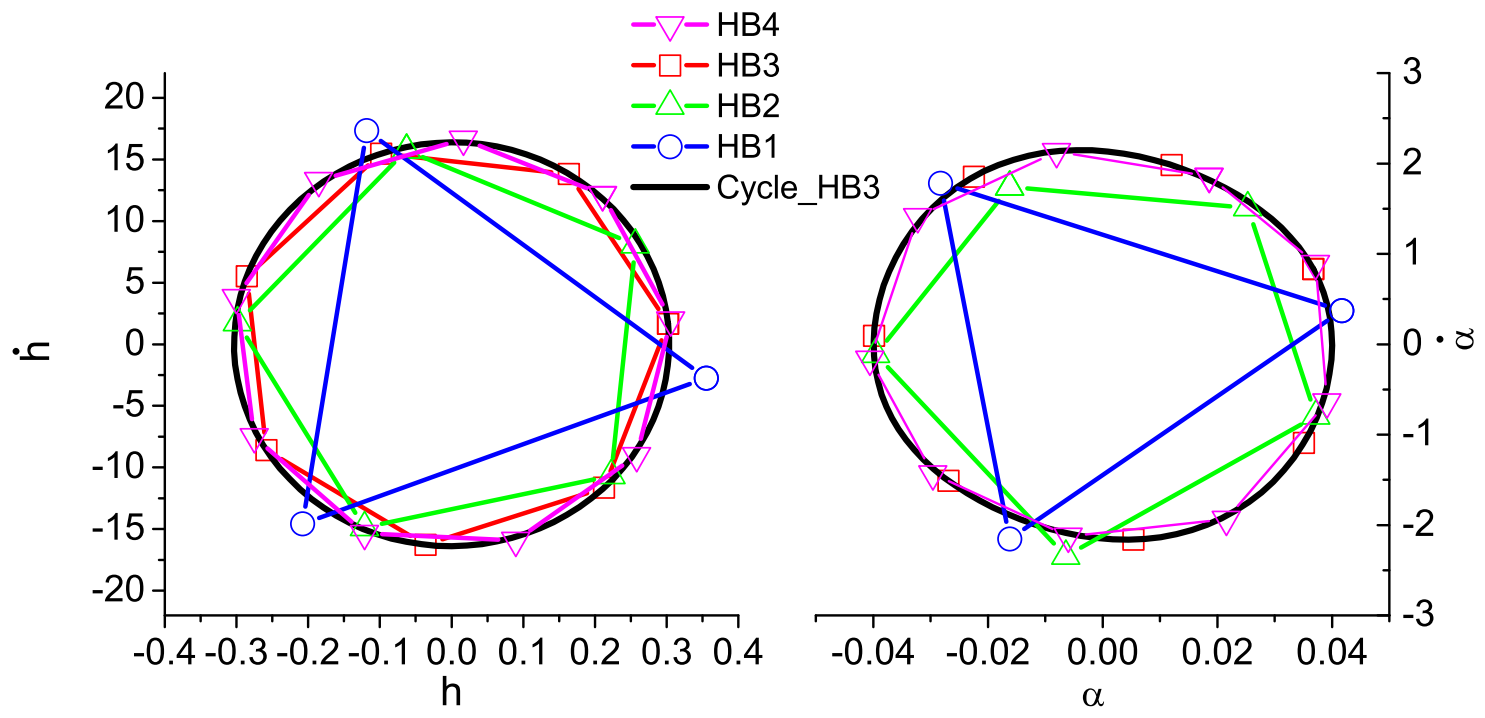

Figure 11. Position - Velocity diagram at LCO condition - $V_{s}=0.80$ - Harmonic Convergence
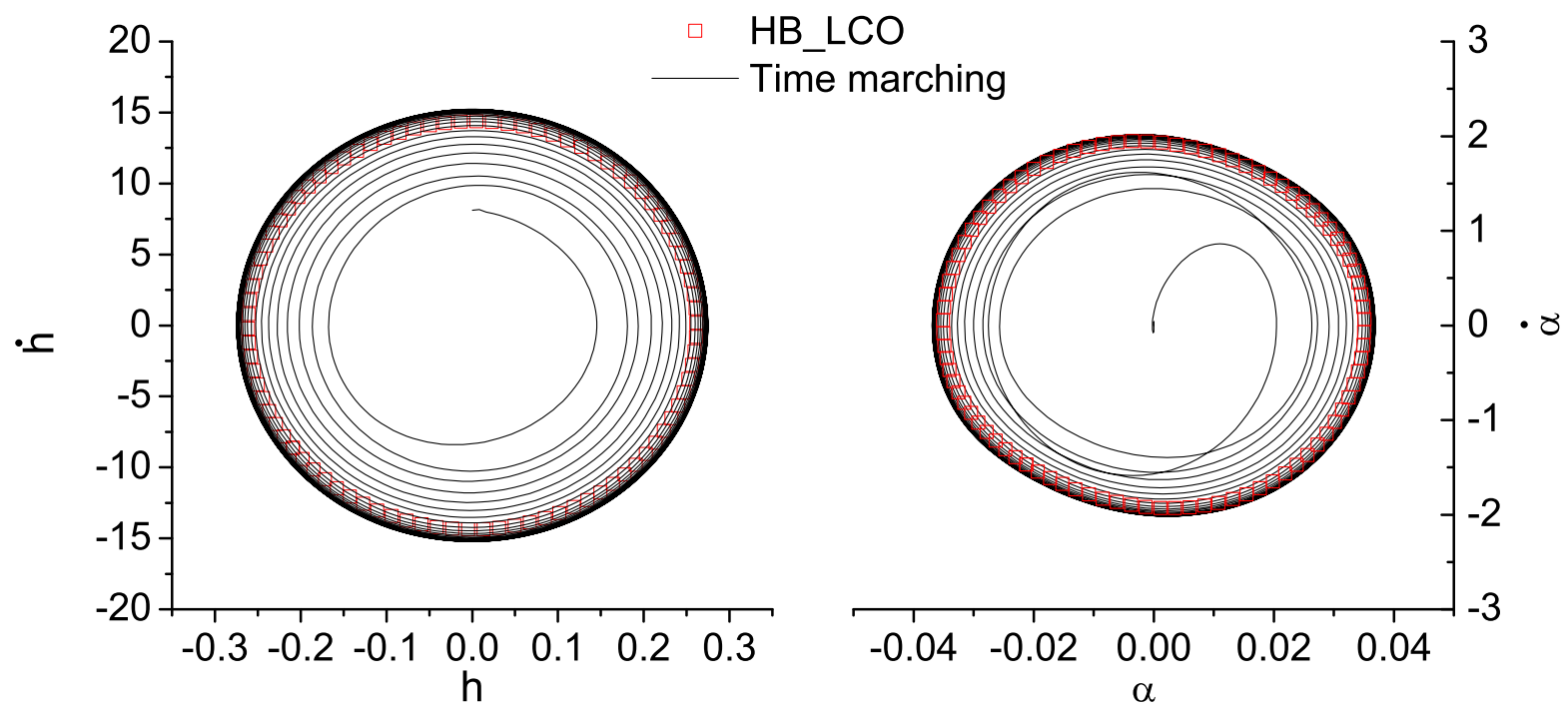

Figure 12. Comparison between A-HB and time marching LCO solutions $-V_{s}=0.725$ 


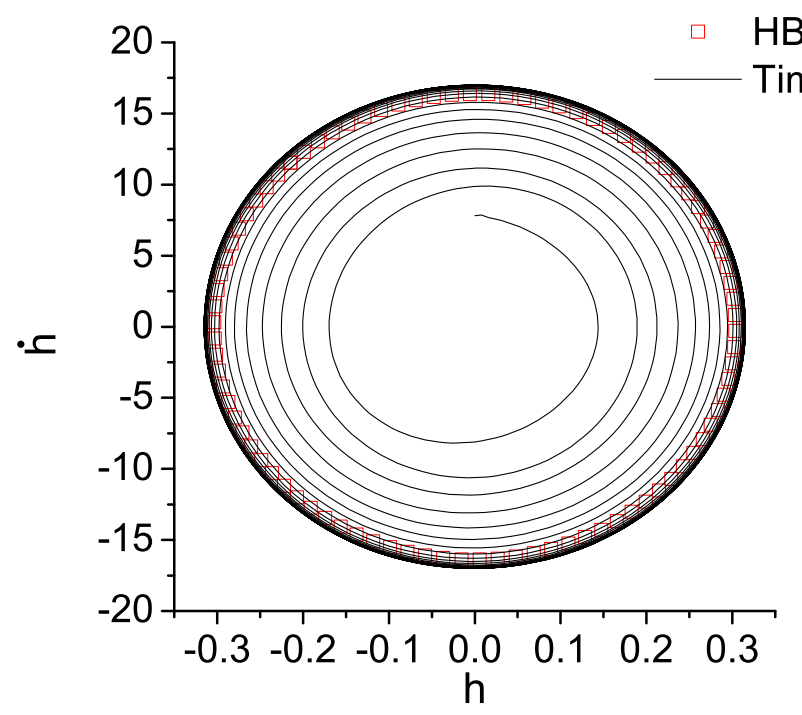

HB_LCO

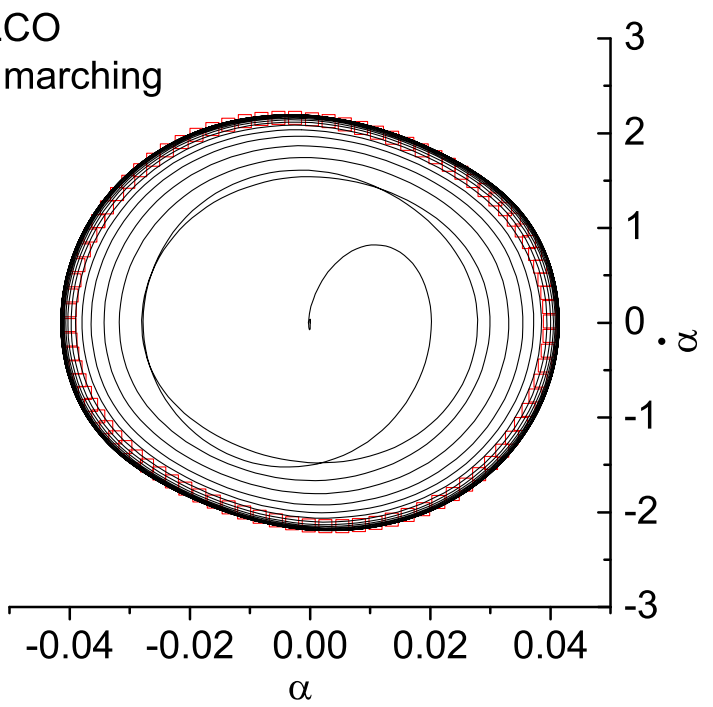

Figure 13. Comparison between A-HB and time marching LCO solutions $-V_{s}=0.80$
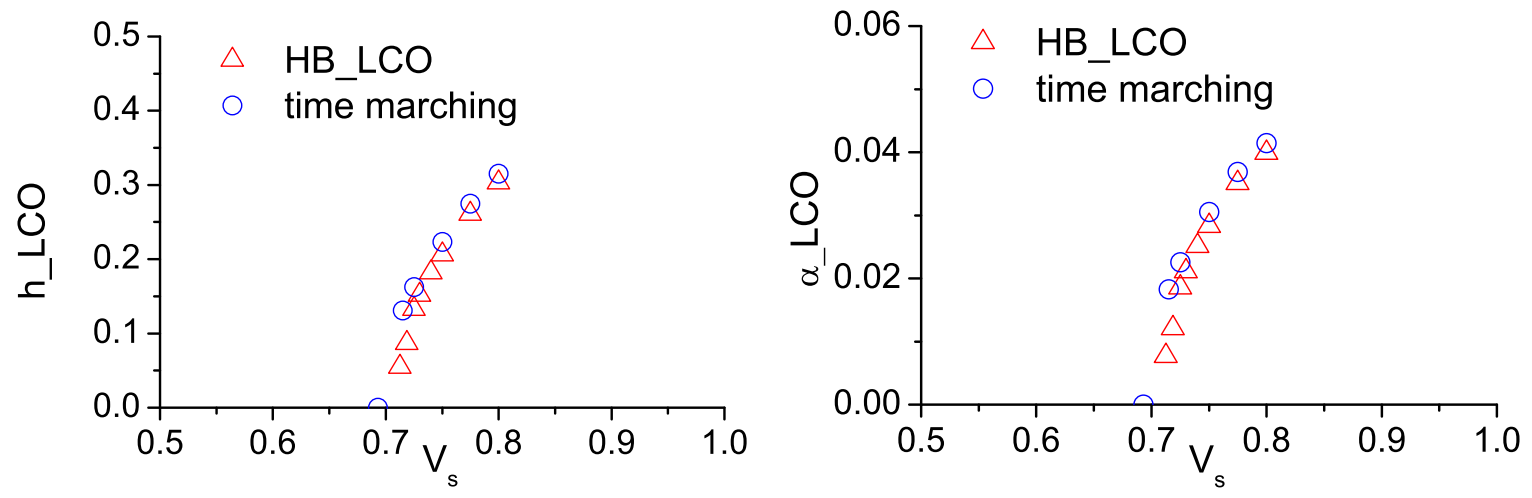

(a) LCO Amplitude vs. Velocity

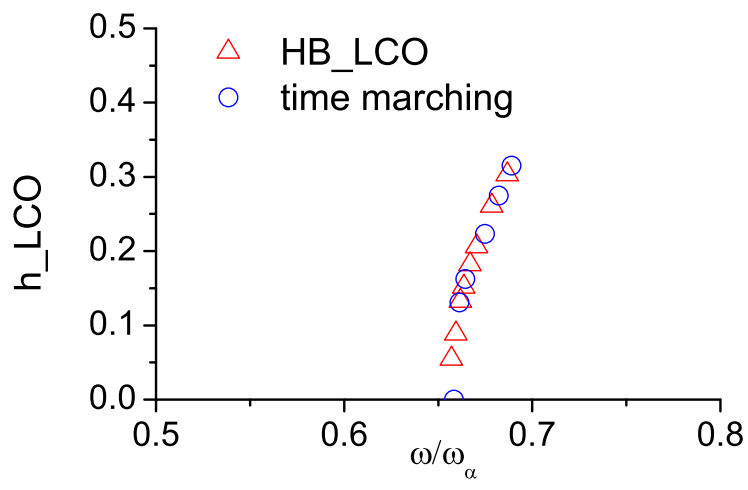

(b) LCO Amplitude vs. Velocity

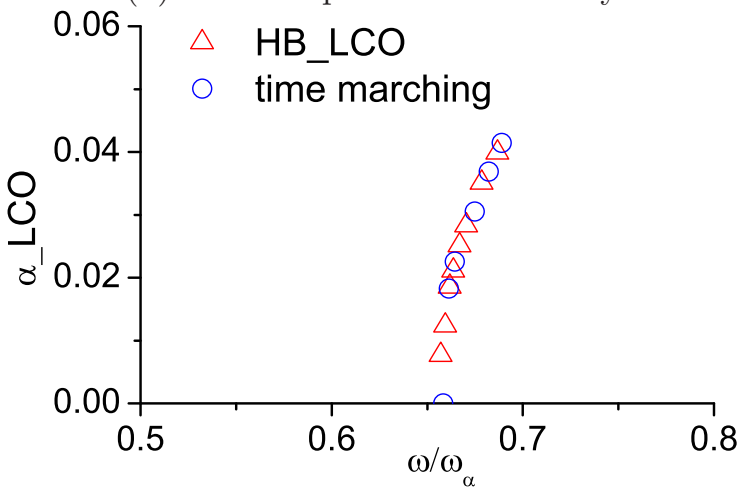

(c) LCO Amplitude vs. Frequency Ratio

(d) LCO Amplitude vs. Frequency Ratio

Figure 14. LCO Amplitude Comparison between A-HB and time marching methods. 


\section{Delta Wing}

To further exercise the proposed method, a cropped-delta wing test case is explored. The wing plan form is shown in fig.15, the wing has a leading edge sweep angle of approximately $16^{\circ}$, and a span of just under $4 \mathrm{~m}$. The wing uses a NACA 65A004 aerofoil. An O-H type grid, shown in fig.16-b), was employed for the CFD calculations, a CFD flowfied solution at the nominal conditions of this test case $\left(M_{\infty}=0.91 ; \alpha=0^{\circ}\right)$ is shown in fig.16-c), where a shock-wave is visible across the span of the wing.

To investigate LCOs, a structural model is coupled with the CFD mesh. The structural model is built in $\mathrm{MSc} /$ Nastran, using 2D shell elements; the wing material is based on the AGARD 445.6 wing, ${ }^{25}$ this results in the first four normal modes retained for this analysis

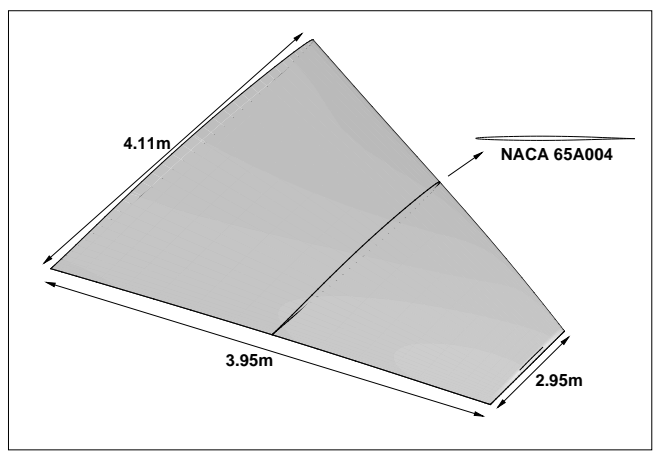

Figure 15. Delta Wing Geometry and Dimensions to have frequencies in the range of $4 \mathrm{~Hz}-30 \mathrm{~Hz}$. The mode shapes and natural frequencies are given in fig.17. Infinite Plate Spline (IPS) is used to extrapolate structural modal displacements from the CSD model to the CFD grid, as shown in fig.17. The starting point of this LCO investigation is the flutter boundary, even-though is not always necessary, since proposed HB method should deliver a trivial solution below the flutter boundary. As before, Volterra series along with the ERA method is employed to construct a linear CFD model coupled with the structural model to form a $808^{\text {th }}$ ROM, as shown in fig.18. The dynamic pressure corresponding to the onset of flutter at $M_{\infty}=0.91, \alpha=0^{\circ}$, predicted by the ROM is $q=0.759 q_{s l}$, where $q_{s l}$ is the dynamic pressure at sea level conditions. The CFD response is included for comparison with the ROM results in fig.18, the ROM is able to replicate the CFD accurately at flutter conditions.

The initial disturbances for the LCO prediction, in modal coordinates and for each mode are: $[1,0.5,0.1,0.1]^{T}$, and the initial reduced frequency is: $\kappa=0.07$. Figure 19 shows the convergence of AHB method for the LCO frequency and displacement. The frequency converges within 250 iterations, with the LCO amplitude requiring a further 50 iterations to reach its final value. The wing undergoes significant

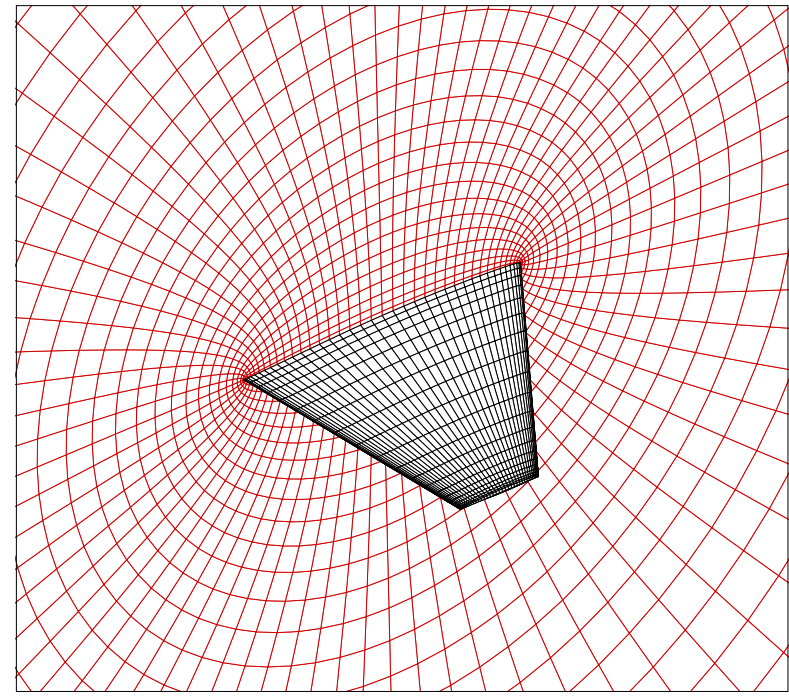

a-) CFD grid $-81 \times 41 \times 41$

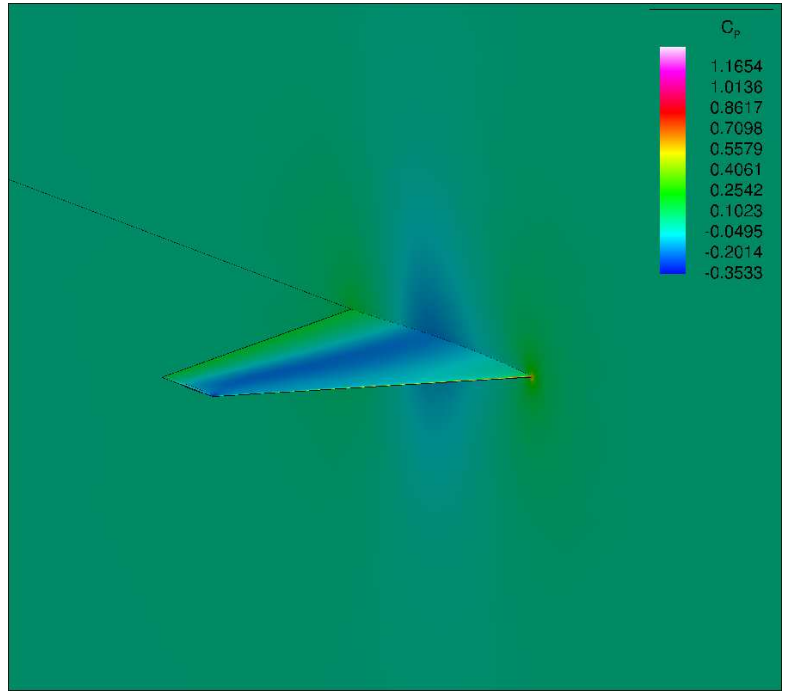

b-) Pressure contours, $M_{\infty}=0.91 ; \alpha=0^{\circ}$

Figure 16. Delta Wing CFD model - the grid contains $81 \times 41 \times 41$ points, circumferential, radial and span-wise direction, respectively 


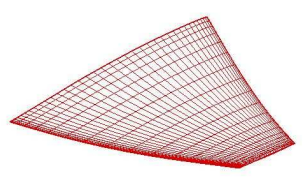

Mode $1-3.94 H z$

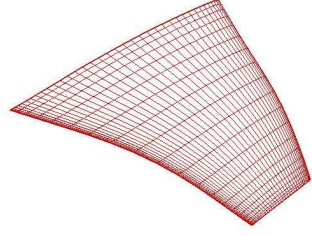

Mode $2-12.88 H z$

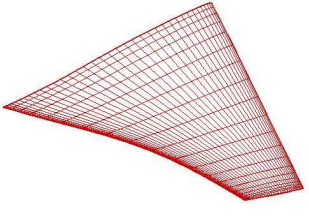

Mode 3 - 15.82

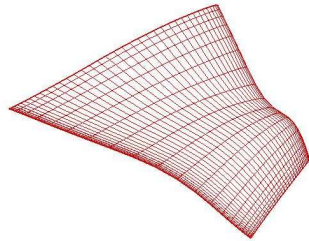

Mode $4-27.56 H z$

Figure 17. Structural modes projected onto the CFD grid.

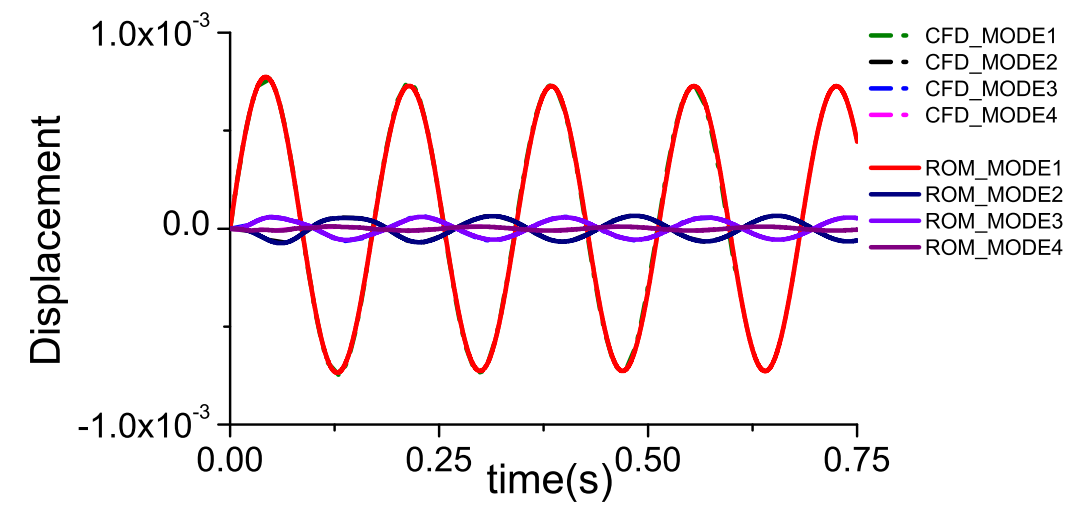

Figure 18. Delta Wing Flutter response with Volterra Series $808^{\text {th }}$ ROM

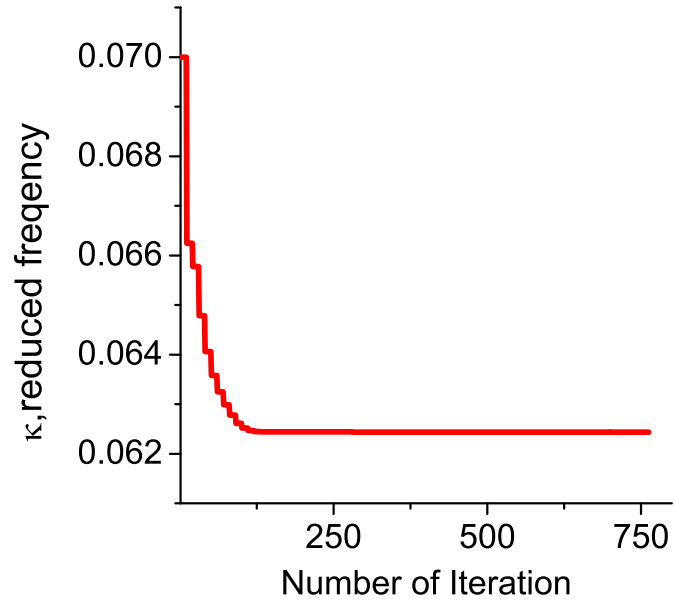

LCO Frequency Convergence

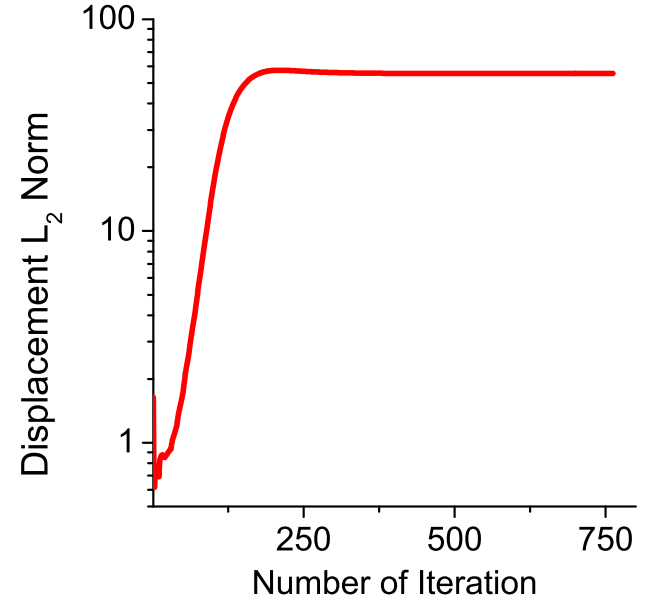

LCO Amplitude Convergence

Figure 19. Delta Wing LCO Response Convergence with A-HB Method 
oscillations at the wing tip, as demonstrated by figs.20 and 21. Two test cases at different free-stream pressures are conducted. Comparison of LCO responses using the A-HB method against time marching results are shown in fig.21, here $\eta_{1}$ and $\eta_{2}$ correspond to points at the wing tip's leading and trailing edges, respectively. The flowfied shown in fig. 20 shows the shock strength increasing as the tip moves downwards and a significant decrease in pressure at the tip's leading edge, as the wing moves upwards, the tip's leading edge pitches nose down and the shock on the upper surface vanishes. Time-marching results required a time step of $10^{-5}$ to converge the cycle amplitude, taking 8.5 days on a single core. The current method is able to predict the LCO conditions accurately using 1 harmonic, reducing the computational time to 8 hours.

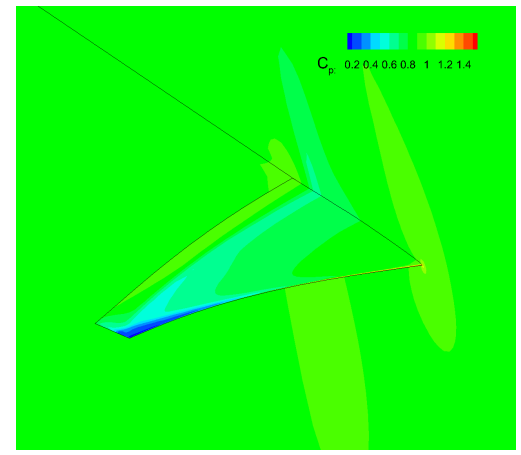

(a) sub-level 1

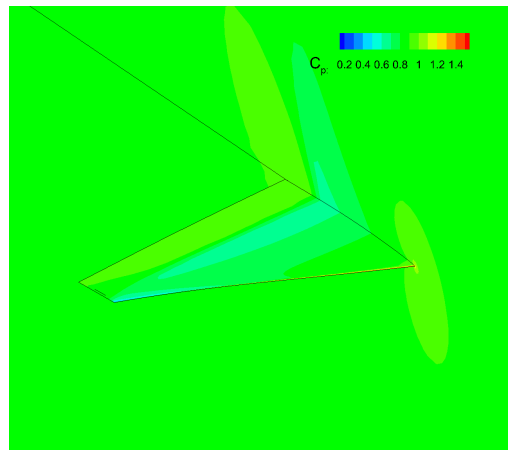

(b) sub-level 2

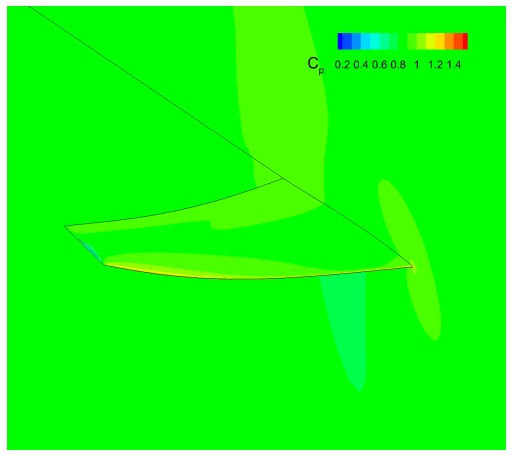

(c) sub-level 3

Figure 20. Delta Wing Pressure Contours snapshots during LCO cycle, $q=0.900 q_{s l}$

\section{Conclusion}

This paper presents a new formulation to predict transonic LCOs type of instabilities for aerofoils and fixed wings, using an Aeroelastic Harmonic Balance methodology. A CFD and CSD system of equations are coupled and solved using a nonlinear Harmonic Balance method. To determine LCO conditions, the coupled system is driven to convergence by updating the aeroelastic system's frequency of motion considering the influence of aerodynamic forces. The new method, shows promising results in predicting LCO amplitudes and frequencies for multiple-degrees-of-freedom aeroelastic systems, including 2D aerofoils and a delta wing at transonic conditions. Results show at least an order of magnitude reduction in computational time with respect to conventional time marching methods, without compromising accuracy.

\section{Acknowledgements}

This work was sponsored by the United Kingdom Engineering and Physical Sciences Research Council (grant number EP/K005863/1). The authors gratefully acknowledged this support.

\section{References}

${ }^{1}$ Noll, T. E., Brown, J. M., Perez-Davis, M. E., Ishmael, S. D., Tiffany, G. C., and Gaier, M., "Investigation of the Helios Prototype Aircraft Mishap," Tech. rep., NASA, 2004.

${ }^{2}$ Bunton, R. and Denegri, C., "Limit Cycle Characteristics of Fighter Aircraft," Journal of Aircraft, Vol. 37, No. 5, 2000, pp. 916-918.

${ }^{3}$ Thomas, J., Dowell, E., Hall, K., and Denegri, C., "Modeling limit cycle oscillation behavior of the F-16 fighter using a harmonic balance approach," Tech. rep., AIAA, 2004, Presented at the AIAA/ASME/ASCE/AHS/ASC Structures, Structural Dynamics, and Materials Conference.

${ }^{4}$ Beran, P., Knot, N., Eastep, F., Synder, R., and Zweber, J., "Numerical Analysis of Store-Induced Limit Cycle Oscillarion," Journal of Aircraft, Vol. 41, No. 6, 2004, pp. 1315-1326.

${ }^{5}$ Lieu, T., Farhat, C., and Lesoinne, M., "Reduced-order fluid/structure modeling of a complete aircraft configuration," Computer Methods in Applied Mechanics and Engineering, Vol. 195, No. 41-43, 2006, pp. 5730-5742, John H. Argyris Memorial Issue. Part $\{\mathrm{II}\}$.

${ }^{6}$ Silva, W., "Identification of Nonlinear Aeroelastic Systems Based on the Volterra Theory: Progress and Opportunities," Nonlinear Dynamics, Vol. 39, No. 1-2, pp. 25-62. 

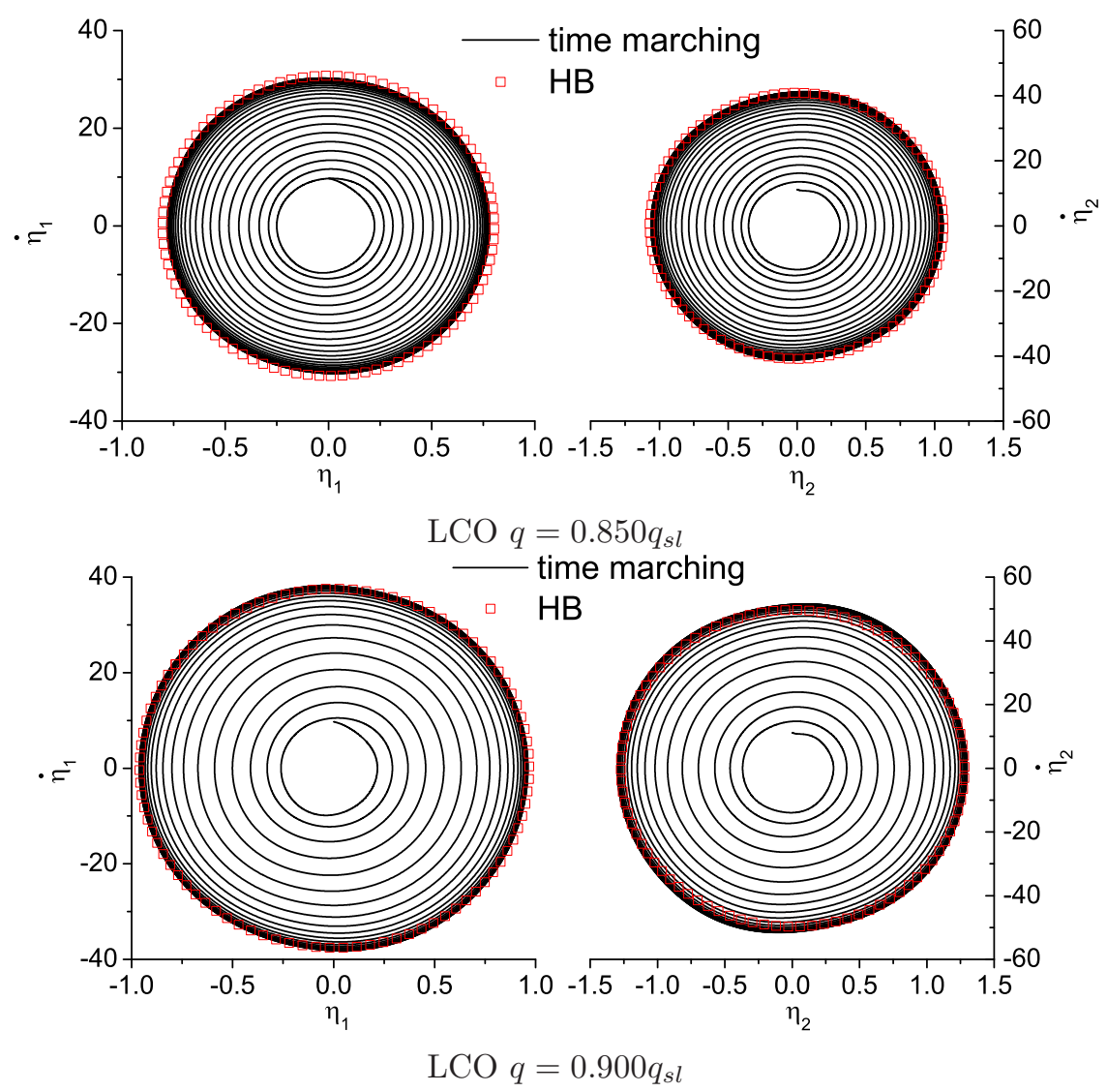

Figure 21. Delta Wing LCO Response with A-HB Method 
${ }^{7}$ Lucia, D., Beran, P., and Silva, W., "Aeroelastic System Development Using Proper Orthogonal Decomposition and Volterra Theory," AIAA Journal, Vol. 42, No. 2, 2005, pp. 509-518.

${ }^{8}$ Munteanu, S., Rajadas, J., Nam, C., and Chattopadhyay, A., "Reduced-order model approach for aeroelastic analysis involving aerodynamic and structural nonlinearities," AIAA Journal, Vol. 43, No. 3, 2005, pp. 560-571.

${ }^{9}$ Yao, W. and Liou, M.-S., "Reduced-Order Modeling for Limit-Cycle Oscillation Using Recurrent Artificial Neural Network," Tech. rep., AIAA, Sep 2012, Presented at the 14th AIAA/ISSMO Multidisciplinary Analysis and Optimization Conference.

${ }^{10}$ Badcock, K. and Woodgate, M., "Fast Prediction of Transonic Aeroelastic Stability and Limit Cycles," AIAA Journal, Vol. 45, No. 6, 2007, pp. 1370-1381.

${ }^{11}$ Badcock, K. J., Khodaparast, H., Timme, S., and Mottershead, J., "Calculating the Influence of Structural Uncertainty on Aeroelastic Limit Cycle Response," AIAA paper 2011-1741, AIAA, 2011, Presented at the 52nd AIAA/ASME/ASCE/AHS/ASC Structures, Structural Dynamics, and Materials Conference.

${ }^{12}$ Hall Kenneth C., Thomas Jeffrey P., and Clark W. S., "Computation of Unsteady Nonlinear Flows in Cascades Using a Harmonic Balance Technique," AIAA Journal, Vol. 40, No. 5, 2002, pp. 879-886, doi: 10.2514/2.1754.

${ }^{13}$ Gopinath, A., Beran, P., and Jameson, A., "Comparative Analysis of Computational Methods for Limit-Cycle Oscillations," 2006, presented at 47th AIAA/ASME/ASCE/AHS/ASC Structures, Structural Dynamics, and Materials Conference.

${ }^{14}$ Woodgate, M. and Badcock, K., "Implicit Harmonic Balance Solver for Transonic Flow with Forced Motions," AIAA Journal, Vol. 47, No. 4, 2009, pp. 893-901.

${ }^{15}$ Blanc, F., Roux, F.-X., and Jouhaud, J.-C., "Harmonic-Balance-Based Code-Coupling Algorithm for Aeroelastic Systems Subjected to Forced Excitation," AIAA Journal, Vol. 48, No. 11, 2010, pp. 2472-2481.

${ }^{16}$ Woodgate, M. and Barakos, G., "Implicit Computational Fluid Dynamics Methods for Fast Analysis of Rotor Flows," AIAA Journal, Vol. 50, No. 6, 2012, pp. 1217-1244.

${ }^{17}$ Ekici, K. and Hall, K., "Harmonic Balance Analysis of Limit Cycle Oscillations in Turbomachinery," AIAA Journal, Vol. 49, No. 7, 2011, pp. $1478-1487$.

${ }^{18}$ Sicot Frédéric, Gomar Adrien, Dufour Guillaume, and Dugeai Alain, "Time-Domain Harmonic Balance Method for Turbomachinery Aeroelasticity," AIAA Journal, Vol. 52, No. 1, 2014, pp. 62-71, doi: 10.2514/1.J051848.

${ }^{19}$ Roe, P., "Approximate Riemann Solvers, Parameter Vectors, and Difference Schemes," Journal of Computational Physics, Vol. 43, No. 2, 1981, pp. 357-372.

${ }^{20}$ van Leer, B., "Towards the ultimate conservative difference scheme. II. Monotonicity and conservation combined in a second-order scheme," Journal of Computational Physics, Vol. 14, No. 4, 1974, pp. 361-370.

${ }^{21}$ Thomas, J., Dowell, E., and Hall, K., "Nonlinear Inviscid Aerodynamic Effects on Transonic Divergence, Flutter, and Limit-Cycle Oscillations," AIAA Journal, Vol. 40, No. 4, 2002, pp. 638-646.

${ }^{22}$ Davis, S., "NACA 64A010 (NASA Ames Model) Oscillatory Pitching," Report 702, AGARD, 1982.

${ }^{23}$ McMullen, M., Jameson, A., and Alonso, J., "Demonstration of Nonlinear Frequency Domain Methods," AIAA Journal, Vol. 44, No. 7, 2006, pp. 1428-143.

${ }^{24}$ Pierce, N. and Alonso, J., "Efficient Computation of Unsteady Viscous Flows by an Implicit Preconditioned Multigrid Method," AIAA Journal, Vol. 36, No. 3, 1998, pp. 401-408.

${ }^{25}$ Yates, E., "AGARD standard aeroelastic configurations for dynamic response. Candidate configuration I.-wing 445.6," Nasa-tm-100492, Aug. 1987. 